\title{
Water delivery by pebble accretion to rocky planets in habitable zones in evolving disks
}

\author{
Shigeru Ida (井田茂 $)^{1}$, Takeru Yamamura (山村 武 $)^{2}$, and Satoshi Okuzumi (奥住 聡 $)^{2}$ \\ ${ }^{1}$ Earth-Life Science Institute, Tokyo Institute of Technology, Meguro-ku, Tokyo 152-8550, Japan \\ e-mail: ida@elsi.jp \\ ${ }^{2}$ Department of Earth and Planetary Sciences, Tokyo Institute of Technology, Meguro-ku, Tokyo 152-8551, Japan
}

Received 1 November 2018 / Accepted 14 January 2019

\begin{abstract}
Context. The ocean mass of the Earth is only $2.3 \times 10^{-4}$ of the whole planet mass. Even including water in the interior, the water fraction would be at most $10^{-3}-10^{-2}$. Ancient Mars may have had a similar or slightly smaller water fraction. What controlled the amount of water in these planets has not been clear, although several models have been proposed. It is important to clarify the control mechanism to discuss water delivery to rocky planets in habitable zones in exoplanetary systems, as well as that to Earth and Mars in our solar system.

Aims. We consider water delivery to planets by icy pebbles after the snowline inwardly passes planetary orbits. We derive the water mass fraction $\left(f_{\text {water }}\right)$ of the final planet as a function of disk parameters and discuss the parameters that reproduce a small value of $f_{\text {water }}$ comparable to that inferred for the Earth and ancient Mars.

Methods. We calculated the growth of icy dust grains to pebbles and the pebble radial drift with a 1D model, by simultaneously solving the snowline migration and dissipation of a gas disk. With the obtained pebble mass flux, we calculated accretion of icy pebbles onto planets after the snowline passage to evaluate $f_{\text {water }}$ of the planets.

Results. We find that $f_{\text {water }}$ is regulated by the total mass $\left(M_{\text {res }}\right)$ of icy dust materials preserved in the outer disk regions at the timing $\left(t=t_{\text {snow }}\right)$ of the snowline passage of the planetary orbit. Because $M_{\text {res }}$ decays rapidly after the pebble formation front reaches the disk outer edge (at $t=t_{\mathrm{pff}}$ ), $f_{\text {water }}$ is sensitive to the ratio $t_{\text {snow }} / t_{\text {pff }}$, which is determined by the disk parameters. We find $t_{\text {snow }} / t_{\text {pff }}<10$ or $>10$ is important. By evaluating $M_{\text {res }}$ analytically, we derive an analytical formula of $f_{\text {water }}$ that reproduces the numerical results. Conclusions. Using the analytical formula, we find that $f_{\text {water }}$ of a rocky planet near 1 au is similar to the Earth, i.e., $\sim 10^{-4}-10^{-2}$, in disks with an initial disk size of 30-50 au and an initial disk mass accretion rate of $\sim\left(10^{-8}-10^{-7}\right) M_{\odot} \mathrm{yr}^{-1}$ for disk depletion timescale of approximately a few M yr. Because these disks may be median or slightly compact/massive disks, our results suggest that the water fraction of rocky planets in habitable zones may often be similar to that of the Earth if icy pebble accretion is responsible for water delivery.
\end{abstract}

Key words. planets and satellites: formation - planets and satellites: terrestrial planets - protoplanetary disks

\section{Introduction}

Earth-size planets are being discovered in habitable zones (HZs) in exoplanetary systems. These HZs are defined as a range of orbital radius, in which liquid water can exist on the planetary surface if $\mathrm{H}_{2} \mathrm{O}$ exists there. However, as long as equilibrium temperature is concerned, $\mathrm{H}_{2} \mathrm{O}$ ice grains condense only well beyond the HZs, because the gas pressure of protoplanetary disks is many orders of magnitude lower than that of planetary atmosphere and the condensation temperature is considerably lower than that at $1 \mathrm{~atm}$. Hereafter, we simply call $\mathrm{H}_{2} \mathrm{O}$ in a solid/liquid phase "water". For the Earth, a volatile supply by the gas capture from the disk is ruled out because observed values of rare-Earth elements are too low in the Earth to be consistent with the disk gas capture (e.g., Brown 1949). Therefore, the water in the Earth would have been delivered from the outer regions of the disk during planet formation.

One possible water delivery mechanism to Earth is the inward scattering of water-bearing asteroids by Jupiter (e.g., Raymond et al. 2004). If this is a dominant mechanism of water delivery, the amount of delivered water is rather stochastic and depends on configurations of giant planets in the planetary systems. If water is not delivered, a rocky planet in a HZ may not be able to be an actual habitat. On the other hand, too much water creates a planet without continents, where the supply of nutrients may not be as effective as in the Earth. The oceans of the Earth comprise only $0.023 \%$ by weight and this amount enables oceans and continents to coexist. The mantle may preserve water in the transition zone with a comparable amount of ocean (e.g., Bercovici \& Karato 2003; Hirschmann 2006; Fei et al. 2017), while the core could have $\mathrm{H}$ equivalent to $2 \%$ by weight of $\mathrm{H}_{2} \mathrm{O}$ of the Earth (Nomura et al. 2014). However, the original water fraction of the Earth would still be very small $\left(\sim 10^{-4}-10^{-2}\right)$, even with the possible water reservoir in the interior because neither stellar irradiation at $\sim 1$ au (Machida \& Abe 2010) nor giant impacts (Genda \& Abe 2005) can vaporize the majority of the water from the gravitational potential of Earth. We note that it is inferred that Mars may have subsurface water of $10^{-4}-10^{-3}$ of Mars mass (e.g., di Achille \& Hynek 2010; Clifford et al. 2010; Kurokawa et al. 2014). From the high D/H ratio observed in the Venus atmosphere, early Venus may also have had oceans of the fraction $10^{-5}-10^{-3}$ and lost the $\mathrm{H}_{2} \mathrm{O}$ vapor through runaway greenhouse effect (e.g., Donahue et al. 1982; Greenwood et al. 2018). The order of water fraction looks similar at least between the Earth and ancient Mars. Although the estimated total mass fraction of water in the Earth and Mars has relatively 
large uncertainty ranging from $10^{-4}$ to $10^{-2}$, the range is still much smaller than the dispersion predicted by the water-bearing asteroid collision model; this model ranges from $10^{-5}$ to $10^{-1}$, depending on the formation timing, history of orbital migration/eccentricity evolution of gas giant planets, and the original surface density of planetesimals (e.g., Morbidelli et al. 2000; Lunine et al. 2003; Raymond et al. 2004; O'Brien et al. 2014; Matsumura et al. 2016). It is not clear if the similar orders of water fraction between the Earth and Mars is just a coincidence.

Sato et al. (2016) investigated water delivery by icy pebble accretion. Pebble accretion has been proposed as a new mode of planet accretion (e.g., Ormel \& Klahr 2010; Lambrechts \& Johansen 2012). Radiative transfer calculations for viscous accretion disk models show that the water snowline at $\sim 170 \mathrm{~K}$ may migrate to inside $1 \mathrm{au}$ with the grain opacity $\gtrsim \mathrm{mm}$ size, when the disk accretion rate is $\dot{M}_{\mathrm{g}} \lesssim 10^{-8} M_{\odot} \mathrm{yr}^{-1}$ (e.g., Garaud \& Lin 2007; Min et al. 2011; Oka et al. 2011), which is a typical value of $\dot{M}_{\mathrm{g}}$ of classical T Tauri stars (Hartmann et al. 1998). After the snowline inwardly passes a planetary orbit, icy pebbles can be accreted by the planet. In situ ice condensation near the planet orbit is unlikely because the disk gas there was once in the outer region before it migrated to the inner region and icy components have been already condensed to icy grains and subtracted in the outer region (Morbidelli et al. 2016; Sato et al. 2016). Sato et al. (2016) calculated the time evolution of icy pebble mass flux, the solid surface density in the disk, and the growth of a hypothetical planet at 1 au by icy pebble accretion with a $1 \mathrm{D}$ model. They found that pebble accretion is so efficient that the water fraction of the planet rapidly increases after the snowline passage. They assumed a static disk and artificially set the timings of snowline passage and removal of the disk that truncates pebble accretion. They found that the water mass fraction of the final planet is very sensitive to these timings and it is zero or more than 0.1 in many cases. The modest water mass fraction of $10^{-4}-10^{-2}$ is possible only if the disk is compact $(<100 \mathrm{au})$ and the snowline passage at $1 \mathrm{au}$ later than 2-4 M yr after icy dust growth starts, which could be a narrow window of the parameters.

The sensitive dependence of the final water fraction requires that the snowline migration and the decay of the icy dust surface density must be consistently calculated in an evolving disk. We use the disk evolution model based on the self-similar solution for accretion disks with constant viscosity parameter $\alpha$ (Lynden-Bell \& Pringle 1974) ${ }^{1}$. The snowline migration, disk gas decay, and growth/drift of pebbles and the associated evolution of the icy dust surface density are simultaneously calculated by a $1 \mathrm{D}$ disk evolution model. The growth and drift of pebbles are tracked using the single-size approximation formulated by Ormel (2014) and Sato et al. (2016), which enables us to perform fast calculations and survey broad ranges of parameters. Using the numerical results, we also derive an analytical formula for the final water mass fraction of the planets determined by the disk model parameters.

In Sect. 2, we describe the calculation model that we used. In Sect. 3, the results of numerical simulation are shown. We derive the semi-analytical formula that successfully reproduces the numerical results in Sect. 4. In Sect. 5, using the analytical formula, we study the dependence of the planetary water fraction on the disk and pebble accretion parameters, and discuss the disk parameters to realize the water fraction of $10^{-4}-10^{-2}$, which corresponds to the present Earth and ancient Mars. We

1 The wind-driven disk accretion that has recently been proposed (e.g., Suzuki et al. 2016; Bai et al. 2016) is commented on in Sect. 2.1. show that the disk parameters are not in a narrow window and are rather realized in modest disks. Sections. 6 and 7 provide the discussion and summary.

\section{Method}

\subsection{Gas disk model}

In general, an accretion disk consists of an inner region in which the viscous heating is dominated and an outer region in which irradiation from the host star is dominated. According to Ida et al. (2016), we set the disk midplane temperature for the viscousheating dominated region $\left(T_{\text {vis }}\right)$ and the irradiation dominated region $\left(T_{\text {irr }}\right)$ to be

$T_{\text {vis }} \simeq 130\left(\frac{\alpha}{10^{-2}}\right)^{-1 / 5}\left(\frac{M_{*}}{M_{\odot}}\right)^{3 / 10}\left(\frac{\dot{M}_{\mathrm{g}}}{10^{-8} M_{\odot} \mathrm{yr}^{-1}}\right)^{2 / 5}\left(\frac{r}{1 \mathrm{au}}\right)^{-9 / 10} \mathrm{~K}$,

$T_{\mathrm{irr}} \simeq 130\left(\frac{L_{*}}{L_{\odot}}\right)^{2 / 7}\left(\frac{M_{*}}{M_{\odot}}\right)^{-1 / 7}\left(\frac{r}{1 \mathrm{au}}\right)^{-3 / 7} \mathrm{~K}$,

where $r$ is the distance from the host star, $\dot{M}_{\mathrm{g}}$ is the disk gas accretion rate, which is almost independent of $r$ except in outermost region, $L_{*}$ and $M_{*}$ are the luminosity and mass of the host star, respectively, and $L_{\odot}$ and $M_{\odot}$ are their values of the Sun. We adopt the alpha prescription for the disk gas turbulent viscosity (Shakura \& Sunyaev 1973), $v \simeq \alpha h_{\mathrm{g}}^{2} \Omega$, where $h_{\mathrm{g}}$ is the disk gas scale height, defined by $h_{\mathrm{g}}=c_{\mathrm{s}} / \Omega, c_{\mathrm{s}}$ and $\Omega$ are the sound velocity and Kepler frequency, respectively, and $\alpha(<1)$ is a parameter that represents the strength of the turbulence. We use slightly lower $T_{\text {irr }}$ than that in Ida et al. (2016), assuming lower opacity with millimeter-sized dust grains (Oka et al. 2011), because we consider relatively inner disk regions near the snowline and those pebbles which have grown in outer regions and drifted inward. If micron-sized grains are assumed, the same temperature is realized with about ten times smaller $\dot{M}_{\mathrm{g}}$.

One fundamental assumption behind Eq. (1) is that the rate of viscous heating per unit volume scales linearly with the gas density, and is therefore highest at the midplane. This assumption is questioned by magnetohydrodynamic (MHD) models of protoplanetary disks, which show that accretion heating dominantly takes place on the disk surface (Hirose \& Turner 2011). Recently, Mori et al. (2019) investigated this issue using a series of MHD simulations including all nonideal MHD effects, finding that the midplane temperature derived from the simulations is generally lower than that from Eq. (1) because the heat generated near the disk surface can easily be lost through radiation. Mori et al. (2019) have also found that MHD disk winds, which are not included in our disk model, take away $\simeq 30 \%$ of the magnetic energy that would be available for disk heating if the winds were absent. Although there are disk evolution models accounting for the mass and angular momentum loss due to MHD disk winds (e.g., Armitage et al. 2013; Suzuki et al. 2016; Bai et al. 2016; Hasegawa et al. 2017), none of these take into account the two effects mentioned above. For this reason, we opted to adopt the more classical viscous disk model in this study. We note that the viscous accretion model serves as a good approximation of real protoplanetary disks if some hydrodynamical instabilities drive turbulence near the midplane (see Lyra \& Umurhan 2018; Klahr et al. 2018, for recent reviews on hydrodynamic instabilities of protoplanetary disks). 
The gas disk aspect ratios corresponding to Eqs. (1) and (2) are

$\frac{h_{\mathrm{g}, \mathrm{vis}}}{r} \simeq 0.022\left(\frac{\alpha}{10^{-2}}\right)^{-1 / 10}\left(\frac{M_{*}}{M_{\odot}}\right)^{-7 / 20}\left(\frac{\dot{M}_{\mathrm{g}}}{10^{-8} M_{\odot} \mathrm{yr}^{-1}}\right)^{1 / 5}\left(\frac{r}{1 \mathrm{au}}\right)^{1 / 20}$,

$\frac{h_{\mathrm{g}, \mathrm{irr}}}{r} \simeq 0.022\left(\frac{L_{*}}{L_{\odot}}\right)^{1 / 7}\left(\frac{M_{*}}{M_{\odot}}\right)^{-4 / 7}\left(\frac{r}{1 \mathrm{au}}\right)^{2 / 7}$,

where $h_{\mathrm{g}, \mathrm{vis}}$ and $h_{\mathrm{g}, \text { irr }}$ are the gas scale height in the viscousheating dominated and irradiation dominated regions, respectively. Hereafter, we perform simulations with $L_{*}=L_{\odot}$ and $M_{*}=M_{\odot}$, while we retain their dependences in the formulas. The disk region is viscous-heating dominated if $T_{\text {vis }}>T_{\text {irr. }}$. Otherwise, the irradiation dominates. The transition radius between the viscous-heating and irradiation dominated regions is given by

$r_{\text {vis/irr }} \simeq 1\left(\frac{\alpha}{10^{-2}}\right)^{-14 / 33}\left(\frac{L_{*}}{L_{\odot}}\right)^{-20 / 33}\left(\frac{M_{*}}{M_{\odot}}\right)^{31 / 33}\left(\frac{\dot{M}_{\mathrm{g}}}{10^{-8} M_{\odot} \mathrm{y}^{-1}}\right)^{28 / 33} \mathrm{au}$.

Defining the snowline by the location at $\sim 170 \mathrm{~K}, r_{\text {snow }} \simeq$ $\max \left(r_{\text {snow,vis }}, r_{\text {snow,irr }}\right)$, where

$r_{\text {snow,vis }} \simeq 0.74\left(\frac{M_{*}}{M_{\odot}}\right)^{1 / 3}\left(\frac{\alpha}{10^{-2}}\right)^{-2 / 9}\left(\frac{\dot{M}_{\mathrm{g}}}{10^{-8} M_{\odot} \mathrm{yr}^{-1}}\right)^{4 / 9} \mathrm{au}$,

$r_{\text {snow,irr }} \simeq 0.53\left(\frac{L_{*}}{L_{\odot}}\right)^{2 / 3}\left(\frac{M_{*}}{M_{\odot}}\right)^{-1 / 3}$ au.

As $\dot{M}_{\mathrm{g}}$ decreases with time, the snowline migrates inward in the viscous-heating dominated region until $r_{\text {snow,vis }}$ becomes equal to $r_{\text {snow,irr }}\left(\dot{M}_{\mathrm{g}} \gtrsim 5 \times 10^{-9} M_{\odot} \mathrm{yr}^{-1}\right.$ for $\left.\alpha=10^{-2}\right)$. For calculating the evolution of the pebble flux, it may be enough to set up a static disk distribution as in Sato et al. (2016). However, to describe the snowline migration and disk gas depletion (which determines timings of start and termination of the icy pebble supply), we need an evolving gas disk model.

Specific orbital angular momentum is proportional to a square root of orbital radius $r$ and most of disk mass exists in the outer irradiation dominated region. Angular momentum transfer in the entire disk that determines the snowline migration and the entire disk gas depletion is regulated by the evolution of the outer disk region. The region near the snowline is not a hot region where the viscous heating is significantly higher than the irradiation heating (Eqs. (5) and (6)). So, for our purpose, the entire disk evolution model can be approximated by a irradiation dominated disk. In the irradiation dominated disk, the viscosity $v \propto \alpha T r^{3 / 2} \propto \alpha r^{15 / 14}$ (Eq. (2)). Because it is similar to the disk with the viscosity $v \propto r$ with a constant $\alpha$, we adopted the selfsimilar solution with $v \propto r$ by Lynden-Bell \& Pringle (1974) for the dynamical evolution of the entire disk, while we took into account the snowline evolution regulated by time evolution of the viscous heating (Eq. (6)).

In the self-similar solution, well inside the initial characteristic disk size $\left(r_{\mathrm{d}, 0}\right)$, beyond which the surface density decays exponentially, the disk accretion rate is given as a function of time by

$\dot{M}_{\mathrm{g}} \simeq 3 \pi \Sigma_{\mathrm{g}} v \simeq \dot{M}_{\mathrm{g}, 0} \tilde{t}^{-3 / 2}$,

where $\Sigma_{\mathrm{g}}$ is the disk gas surface density, $v$ is the effective viscosity at $r, \tilde{t}=1+t / t_{\mathrm{diff}}, t_{\mathrm{diff}}=r_{\mathrm{d}, 0}^{2} / 3 v_{0}=r_{\mathrm{d}, 0} r / 3 v$ (where $v_{0}$ is the viscosity at $r_{\mathrm{d}, 0}$ ), and $\dot{M}_{\mathrm{g}, 0}$ is the initial disk accretion rate, respectively. Inversely, the time evolution of the surface gas density $\Sigma_{\mathrm{g}}$ is given by

$\Sigma_{\mathrm{g}} \simeq \frac{\dot{M}_{\mathrm{g}, 0}}{3 \pi v} \tilde{t}^{-3 / 2} \exp \left(-\frac{r}{\tilde{t} r_{\mathrm{d}, 0}}\right)$,

where we included the time-dependent exponential taper in the full form of the self-similar solution, because we need to evaluate the total disk mass. Because $v \propto r, \Sigma_{\mathrm{g}} \propto 1 / r$ for $r \ll r_{\mathrm{d}, 0}$.

We add the effect of the photoevaporation with the rate $\dot{M}_{\text {pe }}$, although the standard self-similar solution does not have such a term (also see Sect. 3.1). We are concerned with the region near the snowline. We assume that $r \ll r_{\mathrm{d}, 0}$ and the photoevaporation occurs mainly in the outer region with the constant rate of $\dot{M}_{\mathrm{pe}}$. Accordingly, we set

$\dot{M}_{\mathrm{g}}=\dot{M}_{\mathrm{g}, 0} \tilde{t}^{-3 / 2}-\dot{M}_{\mathrm{pe}}$,

$\Sigma_{\mathrm{g}} \simeq \frac{\dot{M}_{\mathrm{g}, 0} \tilde{t}^{-3 / 2}-\dot{M}_{\mathrm{pe}}}{3 \pi v} \exp \left(-\frac{r}{\tilde{t} r_{\mathrm{d}, 0}}\right)$.

Integrating Eq. (11), the disk mass at $t$ is given by

$M_{\mathrm{g}}(\tilde{t})=\int 2 \pi r \Sigma_{\mathrm{g}}(\tilde{t}) \mathrm{d} r=2 \tilde{t} t_{\mathrm{diff}}\left(\dot{M}_{\mathrm{g}, 0} \tilde{t}^{-3 / 2}-\dot{M}_{\mathrm{pe}}\right)$,

where we use $r / 3 v=t_{\mathrm{diff}} / r_{\mathrm{d}, 0}$. The above equations show that the disk accretion rate $\dot{M}_{\mathrm{g}}$ and the disk gas surface density $\Sigma_{\mathrm{g}}$ quickly decay when $\dot{M}_{\mathrm{g}}$ decreases to the level of $\dot{M}_{\mathrm{pe}}$. This photoevaporation effect avoids long-tail existence of disk gas significantly longer than a few $\mathrm{M}$ yr.

Here, we describe the disks with the parameters, $t_{\text {diff }}$, $\dot{M}_{\mathrm{g}, 0}, \dot{M}_{\mathrm{pe}}$ and $r_{\mathrm{d}, 0}$. The disk depletion timescale $\left(t_{\mathrm{dep}}\right)$ and the disk gas accretion rate onto the host star $\left(\dot{M}_{\mathrm{g}}\right)$ are better constrained by observations than the other parameters, i.e., $t_{\text {dep }} \sim 10^{6}-10^{7}$ yr and $\dot{M}_{\mathrm{g}} \sim\left(10^{-9}-10^{-7}\right) M_{\odot} \mathrm{yr}^{-1}$ for solar-type stars (e.g., Haisch et al. 2001; Hartmann et al. 1998, 2016; Williams \& Cieza 2011). We focus on the systems around solar-type stars. We assume that angular momentum transfer by turbulent viscous diffusion is a major mechanism for disk depletion rather than photoevaporation. We identify $t_{\text {dep }}$ as $t_{\text {diff }}$. We use $\dot{M}_{\mathrm{g}, 0}$, which may be slightly higher than the observed averaged values of $\dot{M}_{\mathrm{g}}$ because we want to set the snowline beyond the orbits of planetary embryos. We perform simulations with $t_{\text {diff }}=10^{6}$ and $3 \times 10^{6} \mathrm{yr}, \dot{M}_{\mathrm{g}, 0}=3 \times 10^{-8} M_{\odot} \mathrm{yr}^{-1}$, and $10^{-7} M_{\odot} \mathrm{yr}^{-1}$. Although $r_{\mathrm{d}, 0}$ is not observationally well constrained, Sato et al. (2016) showed that this parameter is most important for the time evolution of the pebble mass flux. For $r_{\mathrm{d}, 0}$, we use $r_{\mathrm{d}, 0}=30,100$ and $300 \mathrm{au}$, which correspond to the range of the observationally inferred disk size (Williams \& Cieza 2011). We adopt the photoevaporation rate $\dot{M}_{\mathrm{pe}}=10^{-9}$ and $10^{-8} M_{\odot} \mathrm{yr}^{-1}$.

The disk diffusion timescale is

$$
\begin{aligned}
t_{\mathrm{diff}} & \simeq \frac{r_{\mathrm{d} .0}^{2}}{3 v_{0}}=\frac{r_{1} r_{\mathrm{d} .0}}{3 v_{1}}=\frac{r_{1}^{2}}{3 \alpha h_{\mathrm{g}, 1}^{2} \Omega_{\mathrm{K}, 1}} \frac{r_{\mathrm{d} .0}}{r_{1}} \\
& =\frac{1}{6 \pi \alpha}\left(\frac{r_{1}}{h_{\mathrm{g}, 1}}\right)^{2} \frac{r_{\mathrm{d} .0}}{r_{1}} \mathrm{yr} \simeq 10^{2} \alpha^{-1}\left(\frac{r_{\mathrm{d} .0}}{1 \mathrm{au}}\right) \mathrm{yr},
\end{aligned}
$$

where subscript " 1 " expresses values at $1 \mathrm{au}, v_{\mathrm{d} .0}=v\left(r_{\mathrm{d} .0}\right)$ is viscous coefficient at the outer edge of the disk, and $v=v_{1}$. $\left(r / r_{1}\right)$. From this equation, $\alpha$ is calculated as

$\alpha \simeq 10^{-2}\left(\frac{t_{\mathrm{diff}}}{10^{6} \mathrm{yr}}\right)^{-1}\left(\frac{r_{\mathrm{d} .0}}{100 \mathrm{au}}\right)$. 

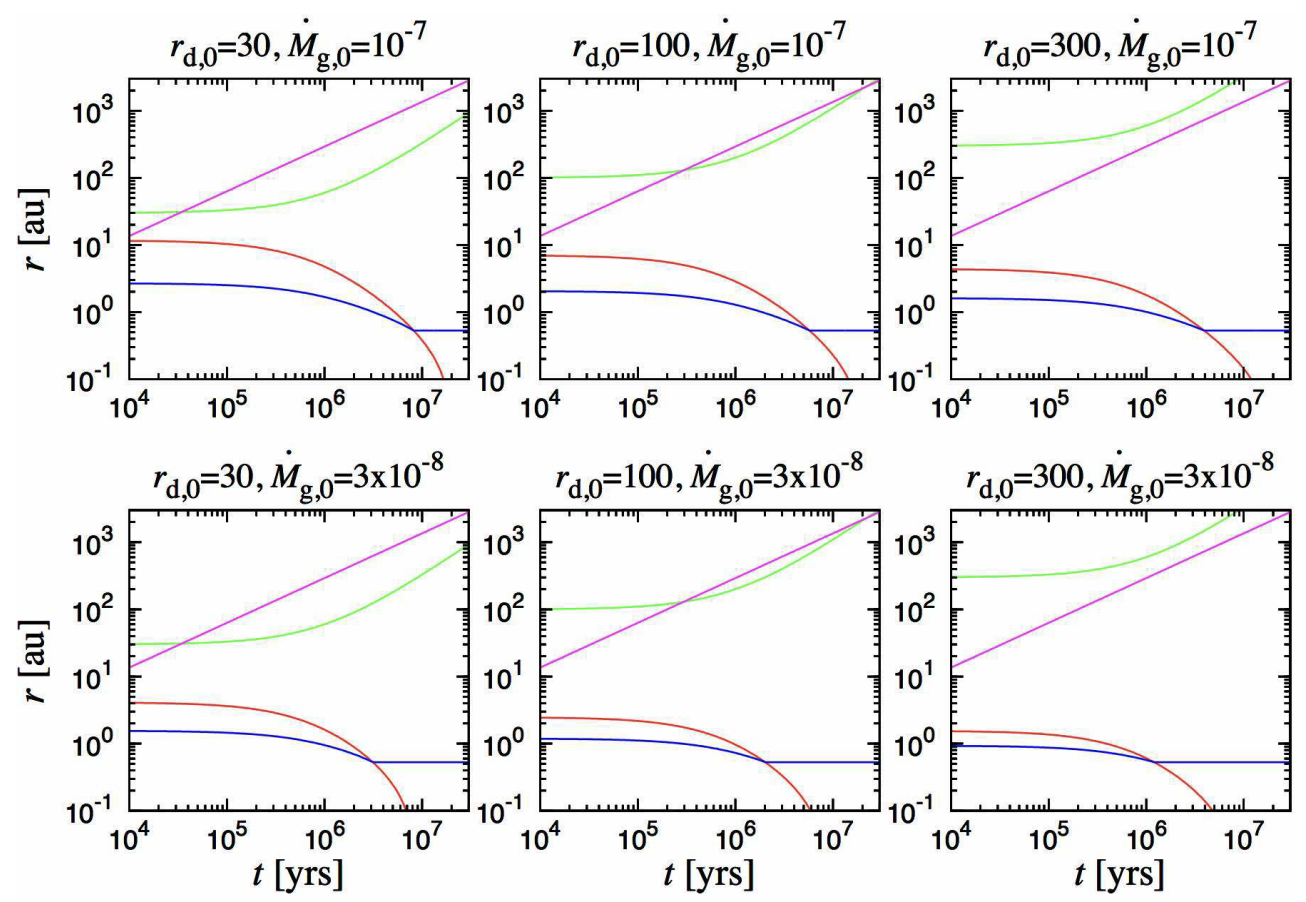

Fig. 1. Time evolution of the transition radius $r_{\text {vis/irr }}$ (Eq. (5); red lines), snowline $r_{\text {snow }}=\max \left(r_{\text {snow,vis }}, r_{\text {snow,irr }}\right)$ (Eqs. (6) and (7); blue lines), disk characteristic radius $\tilde{t} r_{\mathrm{d}, 0}$ (green lines), and pebble formation front $r_{\text {pff }}$ (Eq. (36); magenta lines) for various disk evolution parameters. The parameters, $r_{\mathrm{d}, 0}$ in units of au and $\dot{M}_{\mathrm{g}, 0}$ in units of $M_{\odot} \mathrm{yr}^{-1}$ are labeled in the individual panels. The other parameters are the same for the four panels, i.e., $\dot{M}_{\mathrm{pe}}=10^{-9} M_{\odot} \mathrm{yr}^{-1}$ and $t_{\mathrm{diff}}=10^{6} \mathrm{yr}$.
We note that the value of $\alpha$ that we use in our simulations depends on the choice of the parameters $t_{\mathrm{diff}}$ and $r_{\mathrm{d}, 0}$, but it is within a reasonable range, $\alpha=10^{-3}-3 \times 10^{-2}$. In our formulation for disks, we prefer the setting of the observable valuables, $t_{\text {diff }}$ and $r_{\mathrm{d}, 0}$, within the observationally inferred ranges to a simple assumption of a constant $\alpha$.

In Fig. 1, we show time evolution of the transition radius $r_{\text {vis/irr }}$, the snowline $r_{\text {snow }}$, the disk characteristic radius $\tilde{t} r_{\mathrm{d}, 0}$, and the pebble formation front $r_{\text {pff }}$, which are derived in Sect. 3.1 as Eq. (36), for six typical parameters of disk evolution. In general, $r_{\text {pff }}$ in magenta lines evolves faster than $\tilde{t} r_{\mathrm{d}, 0}$ in the green lines and the pebble flux quickly decays after $r_{\text {pff }}$ exceeds $\tilde{t} r_{\mathrm{d}, 0}$, as we discuss in Sect. 3.1. The snowline $r_{\text {snow }}$ in the blue lines shrinks with time as long as it is in the viscous-heating region (inside of $r_{\text {vis/irr }}$ in the red lines).

\subsection{Dust/pebble evolution model}

We adopt the method with the single size approximation formulated by Ormel (2014) and Sato et al. (2016) to calculate the pebble mass flux. The size distribution of icy particles obtained by a full-size simulation is generally peaky at some size and the peak size depends on $r$. In the single size approximation, icy dust particles have a single size that depends on $r$, corresponding to the peak size.

We briefly summarize the dust evolution model (for more details, see Sato et al. 2016). We set the initial particle surface density as $\Sigma_{\mathrm{p}}=Z_{0} \Sigma_{\mathrm{g}}$. We adopt $Z_{0}=0.01$. The evolution of the dust/pebble surface density $\left(\Sigma_{\mathrm{p}}\right)$ and the peaked mass of the particles $\left(m_{\mathrm{p}}\right)$ are calculated by the equation,

$\frac{\partial \Sigma_{\mathrm{p}}}{\partial t}+\frac{1}{r} \frac{\partial}{\partial r}\left(r v_{r, \mathrm{~d}} \Sigma_{\mathrm{p}}\right)=0$,

$\frac{\partial m_{\mathrm{p}}}{\partial t}+v_{r, \mathrm{~d}} \frac{\partial m_{\mathrm{p}}}{\partial r}=\frac{2 \sqrt{\pi} R_{\mathrm{p}}^{2} \Delta v_{\mathrm{pp}}}{h_{\mathrm{p}}} \Sigma_{\mathrm{p}}$,

where $R_{\mathrm{p}}=\left(3 m_{\mathrm{p}} / 4 \pi \rho_{\text {int }}\right)^{1 / 3}$ is the particle radius, $\rho_{\text {int }}$ is the internal density of icy particles, $v_{r, \mathrm{~d}}$ and $\Delta v_{\mathrm{pp}}$ are the radial and relative velocities of the particles at the midplane, respectively, and $h_{\mathrm{p}}$ is the scale height of the particles given by (e.g. Youdin \& Lithwick 2007)

$h_{\mathrm{p}}=h_{\mathrm{g}}\left(1+\frac{S t}{\alpha} \frac{1+2 S t}{1+S t}\right)^{-1 / 2}$.

The Stokes number defined by $S t=t_{\mathrm{S}} \Omega$ is an important dimensionless number in pebble growth and radial drift, where $t_{\mathrm{s}}$ is the stopping time that represents the timescale of particles's momentum relaxation due to gas drag, given by

$t_{\mathrm{s}}=\left\{\begin{array}{ll}\frac{\rho_{\mathrm{int}} R_{\mathrm{p}}}{\rho_{\mathrm{g}} v_{\mathrm{th}}} & \left(R_{\mathrm{p}}<\frac{9}{4} \lambda_{\mathrm{mfp}} ; \text { Epstein regime }\right) \\ \frac{4 \rho_{\mathrm{int}} R_{\mathrm{p}}^{2}}{9 \rho_{\mathrm{g}} v_{\mathrm{th}} \lambda_{\mathrm{mfp}}} & \left(R_{\mathrm{p}}>\frac{9}{4} \lambda_{\mathrm{mfp}} ; \text { Stokes regime }\right)\end{array}\right.$,

where $v_{\mathrm{th}}=\sqrt{8 k_{\mathrm{B}} T / \pi m_{\mathrm{g}}}$ is the thermal velocity, $\lambda_{\mathrm{mfp}}$ is the mean free path of gas particles, and $m_{\mathrm{g}}$ is the gas molecule mass. The mean free path is expressed by $\lambda_{\mathrm{mfp}}=m_{\mathrm{g}} /\left(\sigma_{\mathrm{mol}} \rho_{\mathrm{g}}\right)$, where $\sigma_{\mathrm{mol}}=2.0 \times 10^{-15} \mathrm{~cm}^{2}$ is the molecular collision cross section.

Relative velocity of dust particles $\Delta v_{\mathrm{pp}}$ is given by

$\Delta v_{\mathrm{pp}}=\sqrt{\left(\Delta v_{\mathrm{B}}\right)^{2}+\left(\Delta v_{\mathrm{r}}\right)^{2}+\left(\Delta v_{\phi}\right)^{2}+\left(\Delta v_{\mathrm{z}}\right)^{2}+\left(\Delta v_{\mathrm{t}}\right)^{2}}$

where $\Delta v_{\mathrm{B}}, \Delta v_{\mathrm{r}}, \Delta v_{\phi}, \Delta v_{\mathrm{z}}$, and $\Delta v_{\mathrm{t}}$ are the relative velocities induced by Brownian motion, radial drift, azimuthal drift, vertical settling, and turbulence, respectively (for detailed expressions, see Sato et al. 2016). We assume perfect sticking for $\Delta v_{\mathrm{pp}}<30 \mathrm{~m} \mathrm{~s}^{-1}$; otherwise, we set the right-hand side of Eq. (16) to be zero.

The radial drift velocity of dust particles is

$v_{\mathrm{r}, \mathrm{d}}=-\frac{2 S t}{1+S t^{2}} \eta v_{\mathrm{K}}$ 
where $v_{\mathrm{K}}=r \Omega_{\mathrm{K}}$ is the Kepler velocity and $\eta$ is a deviation of gas rotation velocity from Kepler velocity, which we set

$\eta=1.1 \times 10^{-3}\left(\frac{r}{1 \mathrm{au}}\right)^{1 / 2}$.

If the effect of the disk gas accretion is taken into account, $2 S t$ in the numerator of Eq. (20) is replaced by $(2 S t+\alpha)$ (e.g., Ida et al. 2016). However, it is neglected here, because the numerical simulation shows $S t \gtrsim 0.1$ for migrating pebbles (Sect. 3.1). The radial drift timescale is

$t_{\text {drift }} \equiv \frac{r}{\left|v_{\mathrm{r}, \mathrm{d}}\right|} \simeq \frac{1}{2 \eta S t \Omega} \simeq 7 \times 10^{4}\left(\frac{S t}{0.1}\right)^{-1}\left(\frac{r}{100 \mathrm{au}}\right)\left(\frac{M_{*}}{M_{\odot}}\right)^{-1 / 2} \mathrm{yr}$.

The pebble mass flux through the disk is given by

$\dot{M}_{\mathrm{peb}}=2 \pi r \Sigma_{\mathrm{p}}\left|v_{r, \mathrm{~d}}\right| \simeq 4 \pi S t \eta r^{2} \Sigma_{\mathrm{p}} \Omega$,

where $\Sigma_{\mathrm{p}}$ is the pebble surface density in the pebble migrating region and we assume $S t^{2} \ll 1$.

\subsection{Pebble accretion onto planets}

We use the same formulas as Sato et al. (2016) for the pebble accretion rate onto planets, assuming that the planets are already large enough for pebble accretion in the "settling regime" (e.g., Ormel \& Klahr 2010; Guillot et al. 2014). Ormel \& Klahr (2010) derived the cross section of pebble accretion as

$\pi b_{\mathrm{set}}^{2} \simeq 4 \pi S t \frac{G M_{\mathrm{pl}}}{\Omega \Delta v}$

where $M_{\mathrm{pl}}$ is the planetary embryo mass and $\Delta v$ is the relative velocity between the embryo and pebbles. The $3 \mathrm{D}$ pebble accretion rate $\dot{M}_{\mathrm{pl}}$ onto the planetary embryo is given by

$$
\begin{aligned}
\dot{M}_{\mathrm{pl}} & \simeq \pi b_{\mathrm{set}}^{2} \rho_{\mathrm{p}} \Delta v \simeq \pi b_{\mathrm{set}}^{2} \frac{\Sigma_{\mathrm{p}}}{\sqrt{2 \pi} h_{\mathrm{p}}} \Delta v \\
& \simeq \frac{r}{\sqrt{2 \pi} h_{\mathrm{p}}} \eta^{-1} \frac{M_{\mathrm{pl}}}{M_{*}} \dot{M}_{\mathrm{peb}} \equiv f_{\mathrm{flt}} \dot{M}_{\mathrm{peb}}
\end{aligned}
$$

where $\rho_{\mathrm{p}}$ is the spatial mass density of the pebbles and we used Eqs. (23) and (24). The parameter $f_{\text {flt }}$ expresses the mass fraction of the accretion flow onto the planet in the pebble flux through the disk before the accretion, which is called a "filtering factor." We note that $f_{\text {fit }}$ does not directly depend on $\Delta v, b_{\text {set }}$, and St.

When $b_{\text {set }}>h_{\mathrm{p}}$, the accretion is $2 \mathrm{D}$ and $\pi b_{\text {set }}^{2} \rho_{\mathrm{p}}$ in Eq. (25) is replaced by $2 b_{\text {set }} \Sigma_{\mathrm{p}}$. Accordingly, a complete filtering factor is

$f_{\mathrm{flt}}=\min \left(\frac{2 r}{\pi b_{\mathrm{set}}}, \frac{r}{\sqrt{2 \pi} h_{\mathrm{p}}}\right) \eta^{-1} \frac{M_{\mathrm{pl}}}{M_{*}}$.

While $f_{\text {flt }}$ depends on $b_{\text {set }}$ in 2D mode, it still does not depend directly on $S t$ and $\Delta v$. In our simulations, we set the planetary embryos with the masses and semimajor axes identical to Venus, Earth, and Mars (eccentricities are set to be zero). In these cases, the relative velocity is given by $\Delta v=(3 / 2) b_{\text {set }} \Omega$ ("Hill regime").
Substituting this into Eq. (24),

$b_{\mathrm{set}} \approx 2 S t^{1 / 3}\left(\frac{M_{\mathrm{pl}}}{3 M_{*}}\right)^{1 / 3} r$.

In the numerical simulations, we use a more general formula including "Bondi regime" and the cutoff parameter $\left(\exp \left[-(S t / 2)^{0.65}\right]\right)$ for large $S t$ cases (Ormel \& Kobayashi 2012). The dependence on $\Delta v$ cancels in the accretion rate both in 2D and 3D cases and $\dot{M}_{\mathrm{pl}}$ does not directly depend on $b_{\text {set }}$ and $S t$ in the 3D case. For a small planet, the accretion is in 3D mode. The accretion becomes $2 \mathrm{D}$ mode when

$$
\begin{aligned}
M_{\mathrm{pl}}>M_{2 \mathrm{D} 3 \mathrm{D}} & \simeq 3\left(\frac{2}{\pi}\right)^{3 / 2} S t^{-1}\left(\frac{S t}{\alpha}\right)^{-3 / 2}\left(\frac{h_{\mathrm{g}}}{r}\right)^{3} M_{*} \\
& \simeq 1.7\left(\frac{M_{*}}{M_{\odot}}\right)^{-5 / 7}\left(\frac{S t}{0.1}\right)^{-5 / 2}\left(\frac{\alpha}{10^{-2}}\right)^{3 / 2}\left(\frac{r}{1 \mathrm{au}}\right)^{6 / 7} M_{\oplus} .
\end{aligned}
$$

The filtering factor is given by

$$
f_{\mathrm{flt}} \simeq\left\{\begin{array}{c}
0.017\left(\frac{M_{*}}{M_{\odot}}\right)^{-1}\left(\frac{\alpha}{10^{-2}}\right)^{-1 / 2}\left(\frac{h_{\mathrm{g}} / r}{0.02}\right)^{-1}\left(\frac{S t}{0.1}\right)^{1 / 2}\left(\frac{M_{\mathrm{pl}}}{0.1 M_{\oplus}}\right)\left(\frac{r}{1 \mathrm{au}}\right)^{-1 / 2} \\
{\left[M_{\mathrm{pl}}<M_{2 \mathrm{D} 3 \mathrm{D}} ; 3 \mathrm{D}\right]} \\
0.040\left(\frac{M_{*}}{M_{\odot}}\right)^{-2 / 3}\left(\frac{S t}{0.1}\right)^{-1 / 3}\left(\frac{M_{\mathrm{pl}}}{0.1 M_{\oplus}}\right)^{2 / 3}\left(\frac{r}{1 \mathrm{au}}\right)^{-1 / 2} \\
{\left[M_{\mathrm{pl}}>M_{2 \mathrm{D} 3 \mathrm{D}} ; 2 \mathrm{D}\right],}
\end{array}\right.
$$

where we used Eq. (21).

We take into account the decrease in dust surface density and pebble flux due to pebble accretion onto the planets. After the snowline passage, Eq. (15) is rewritten in the grid, where the planets exist, as

$\frac{\partial \Sigma_{\mathrm{p}}}{\partial t}+\frac{1}{r} \frac{\partial}{\partial r}\left(r v_{\mathrm{r}, \mathrm{d}} \Sigma_{\mathrm{p}}\right)+\frac{\dot{M}_{\mathrm{pl}}}{2 \pi r \Delta r}=0$,

where $\Delta r$ is the radial grid size. After the icy pebble accretion onto the planet starts, the pebble mass flux decreases discontinuously at the planet orbits, according to the accretion onto the planets.

\subsection{Simulation parameters}

The parameters for our simulations are the initial disk characteristic size $r_{\mathrm{d}, 0}$, the diffusion timescale of the disk $t_{\mathrm{diff}}$, the initial disk gas accretion rate $\dot{M}_{\mathrm{g}, 0}$, and the photo-evaporation rate $\dot{M}_{\text {pe }}$. As we explained in Sect. 2.1, the other disk parameters are calculated by these parameters. We perform simulations with

(1) $\dot{M}_{\mathrm{g}, 0}=3 \times 10^{-8}, 10^{-7} M_{\odot} \mathrm{yr}^{-1}$,

(2) $\dot{M}_{\mathrm{pe}}=10^{-9}, 10^{-8} M_{\odot} \mathrm{yr}^{-1}$,

(3) $r_{\mathrm{d}, 0}=30,100,300 \mathrm{au}$,

(4) $t_{\text {diff }}=10^{6}, 3 \times 10^{6} \mathrm{yr}$.

The initial total gas disk mass is given by Eq. (9) as

$$
\begin{aligned}
M_{\mathrm{g}, 0} & =2 t_{\mathrm{diff}}\left(\dot{M}_{\mathrm{g}, 0}-\dot{M}_{\mathrm{pe}}\right) \\
& =0.06\left(\frac{t_{\mathrm{diff}}}{10^{6} \mathrm{yr}}\right)\left(\frac{\dot{M}_{\mathrm{g}, 0}-\dot{M}_{\mathrm{pe}}}{3 \times 10^{-8} M_{\odot} / \mathrm{yr}}\right) M_{\odot},
\end{aligned}
$$

which ranges from $0.06 M_{\odot}$ to $0.6 M_{\odot}$ with the above parameters. Because we start with relatively large $\dot{M}_{\mathrm{g}, 0}$, the initial disk 
mass is relatively large. As we show in Sect. 4, initially massive disks tend to produce dry planets, while small mass disks tend to produce water-rich planets.

The initial dust surface density distribution is simply given by $0.01 \Sigma_{\mathrm{g}, 0}$. With the gas disk evolution, we simultaneously calculate the pebble growth from the dust disk and its migration. The dust disk is depleted by the formation and radial drift of pebbles.

We set Venus, Earth, and Mars analogs in circular orbits with the same masses and semimajor axes as the current Venus, Earth, and Mars in solar system. For the results presented in this work, we assume $M_{*}=M_{\odot}$ and $L_{*}=L_{\odot}$, while we retain the dependence on $M_{*}$ and $L_{*}$ in the equations. For other-mass stars, the dependence of $\dot{M}_{\mathrm{g}, 0}, \dot{M}_{\mathrm{pe}}, r_{\mathrm{d}, 0}$, and $t_{\mathrm{diff}}$ on the stellar mass also have to be considered.

\section{Numerical results}

\subsection{Icy grain/pebble evolution}

We calculate the growth and radial drift of icy grains and pebbles following the method by Sato et al. (2016), which is described in Sect. 2.2. The evolution of icy particles (grains and pebbles) found by simulations of Sato et al. (2016) is summarized as follows:

1. The growth timescale of a particle with mass $m_{\mathrm{p}}$ is well approximated by the simple formula with $\Delta v_{\mathrm{pp}} \simeq \Delta v_{\mathrm{t}}$ in the Epstein regime (also see Takeuchi \& Lin 2005; Brauer et al. 2008) as

$$
t_{\text {grow }}=\frac{m_{\mathrm{p}}}{\mathrm{d} m_{\mathrm{p}} / \mathrm{d} t} \simeq \frac{4}{\sqrt{3 \pi}} Z_{0}^{-1} \Omega^{-1},
$$

where $Z_{0}$ is the initial particle-to-gas ratio in the disk. The timescale of growth from $\mu \mathrm{m}$-sized grains to pebbles by several orders of magnitude in radius is

$$
\begin{aligned}
t_{\text {grow,peb }} & \sim 10 t_{\text {grow }} \\
& \simeq 2 \times 10^{5}\left(\frac{Z_{0}}{10^{-2}}\right)^{-1}\left(\frac{r}{100 \mathrm{au}}\right)^{3 / 2}\left(\frac{M_{*}}{M_{\odot}}\right)^{-1 / 2} \mathrm{yr} .
\end{aligned}
$$

2. The drift timescale becomes shorter as $m_{\mathrm{p}}$ (equivalently, Stokes number $S t$ ) increases (Eq. (22)), while the growth timescale is independent of $m_{\mathrm{p}}$ (Eq. (32)). When $t_{\text {drift }}$ becomes smaller than $t_{\text {grow }}$, the particle drift effectively starts and $\Sigma_{\mathrm{p}}$ starts being sculpted. From Eqs. (22) and (32) with $Z_{0}$ replaced by the particle-to-gas ratio of drifting pebbles $(Z)$, the equilibrium Stokes number of drifting pebbles is

$$
S t \sim \frac{\sqrt{3 \pi}}{8 \eta} Z \sim 0.03\left(\frac{Z_{0}}{10^{-2}}\right)\left(\frac{r}{100 \mathrm{au}}\right)^{-1 / 2},
$$

where we used a typical value of the solid-to-gas ratio of migrating pebble, $Z \sim 0.1 Z_{0}$ (Ida et al. 2016). We adopt $Z_{0}$ as the initial particle-to-gas ratio and adopt $Z_{0}=0.01$ as a nominal value.

3 . The sensitive $r$-dependence of $t_{\text {grow,peb }}$ results in "an insideout formation" of pebbles; formation is earlier in the inner region and the formation front migrates outward (also see Brauer et al. 2008; Okuzumi et al. 2012; Birnstiel et al. 2012; Lambrechts \& Johansen 2014). After the pebble formation front reaches the characteristic disk radius $\left(r_{\mathrm{d}, 0}\right), \Sigma_{\mathrm{p}}$ rapidly

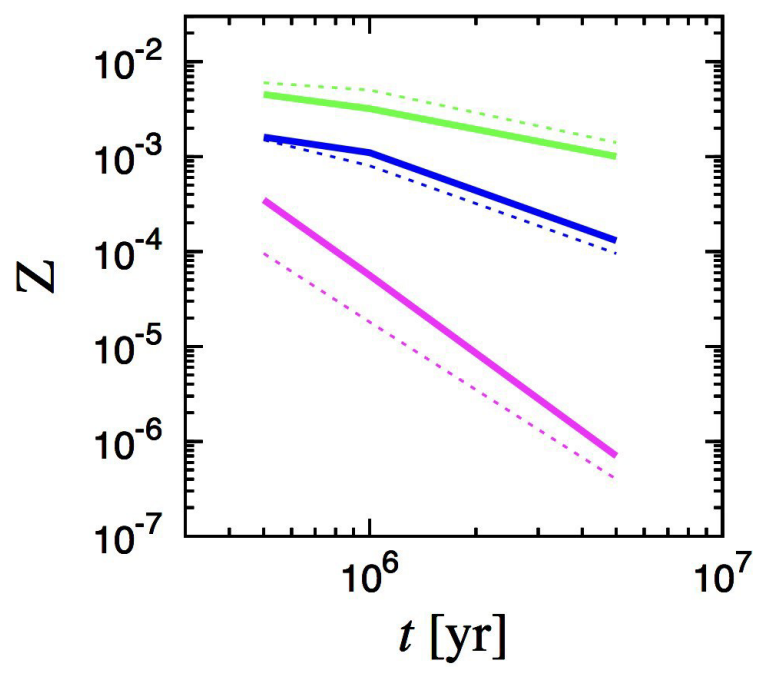

Fig. 2. Time evolution of $Z=\Sigma_{\mathrm{p}} / \Sigma_{\mathrm{g}}$ obtained by the numerical simulation with $\dot{M}_{\mathrm{g}, 0}=10^{-7} M_{\odot} \mathrm{yr}^{-1}, \dot{M}_{\mathrm{pe}}=10^{-9} M_{\odot} \mathrm{yr}^{-1}$, and $t_{\text {diff }}=10^{6} \mathrm{yr}$. The magenta, blue, and green solid lines show $Z$ obtained at $r_{\mathrm{d}, 0}$ by the numerical results of with $r_{\mathrm{d}, 0}=30,100$ and $300 \mathrm{au}$. The dashed lines indicate gradients for individual cases given by Eqs. (37) and (38), where the absolute values of $Z$ are arbitrary.

decays uniformly in the disk because the supply from further outer regions to the region at $r \sim r_{\mathrm{d}, 0}$ is limited.

As we show below, the time $\left(t_{\mathrm{pff}}\right)$ at which the pebble formation front reaches the characteristic disk radius is a very important parameter. The timescale for the pebble formation front to reach the radius of $r_{\mathrm{d}, 0}$ is given with Eq. (33) by

$t_{\mathrm{pff}} \sim 2 \times 10^{5}\left(\frac{Z_{0}}{10^{-2}}\right)^{-1}\left(\frac{r_{\mathrm{d}, 0}}{100 \mathrm{au}}\right)^{3 / 2}\left(\frac{M_{*}}{M_{\odot}}\right)^{-1 / 2} \mathrm{yr}$.

In other words, the pebble formation front radius is given as a function of time $(t)$ by

$r_{\mathrm{pff}} \sim 100\left(\frac{t}{2 \times 10^{5} \mathrm{yr}}\right)^{2 / 3}\left(\frac{Z_{0}}{10^{-2}}\right)^{2 / 3}\left(\frac{M_{*}}{M_{\odot}}\right)^{1 / 3} \mathrm{au}$.

The timescale $t_{\mathrm{pff}}$ is determined only by $r_{\mathrm{d}, 0}$ in the disk parameters. It is independent of the other disk parameters such as $t_{\text {diff }}$ and $\dot{M}_{\mathrm{g}, 0}$.

Once the formation front reaches $r_{\mathrm{d}, 0}$, the supply of solid materials from further outer regions is limited. Accordingly, the pebble formation and drift from there results in the decay of $\Sigma_{\mathrm{p}}$ near $r_{\mathrm{d}, 0}$. The solid surface density at $r<r_{\mathrm{d}, 0}$ also decays because it is contributed from drifting pebbles formed near the formation front. Thus, the decay rate of $\Sigma_{\mathrm{p}}$ for $t>t_{\mathrm{pff}}$ is regulated by the pebble growth near $r_{\mathrm{d}, 0}$, and is approximated as $\partial \Sigma_{\mathrm{p}} / \partial t \sim-\Sigma_{\mathrm{p}} / t_{\mathrm{pff}} \simeq-(\sqrt{3 \pi} / 40)\left(\Sigma_{\mathrm{p}}^{2} / \Sigma_{\mathrm{g}}\right) \Omega$. From this relation, $Z=\Sigma_{\mathrm{p}} / \Sigma_{\mathrm{g}} \propto t^{-1}$ at $t>t_{\mathrm{pff}}$, which is approximated as $Z \propto(1+$ $\left.t / t_{\mathrm{pff}}\right)^{-1}$. However, the numerical results show a faster decrease due to the effect of finite $r_{\mathrm{d}, 0}$ (Sato et al. 2016). The numerically obtained time evolution of $Z$ at $r=r_{\mathrm{d}, 0}$ with $r_{\mathrm{d}, 0}=30,100$ and $300 \mathrm{au}$ is shown in Fig. 2. For $r_{\mathrm{d}, 0}=30,100$, and $300 \mathrm{au}$, $t_{\mathrm{pff}} \simeq 3.3 \times 10^{4}, 2.0 \times 10^{5}$, and $1 \times 10^{6} \mathrm{yr}$, respectively (Eq. (36)) . We fit the numerical results as

$Z \propto\left(1+t / t_{\mathrm{pff}}\right)^{-\gamma}$ 
where

$\gamma=1+\gamma_{2}\left(300 \mathrm{au} / r_{\mathrm{d}, 0}\right)$,

$\gamma_{2} \sim 0.15$.

Because $t_{\mathrm{pff}} \gtrsim t_{\mathrm{diff}}$ and the effect of a finite $r_{\mathrm{d}, 0}$ is not important

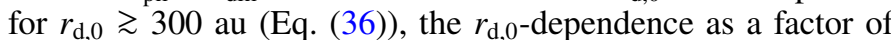
$\left(300 \mathrm{au} / r_{\mathrm{d}, 0}\right)$ is reasonable. Although the value of $\gamma_{2}$ may include an uncertainty due to the disk model and the single-size approximation, we show in Sect. 5 that the predicted function of the water fraction depends only weakly on $\gamma_{2}$.

We note that we assume that collisions between icy pebbles always result in sticking and the pebble size is determined by the drift limit. If the pebble growth is limited by bouncing collisions or collisional fragmentation, $S t$ is determined by the threshold velocity for bouncing or fragmentation, which is lower than the value in Eq. (34), and Eq. (36) depends on the threshold St. It is a widely accepted idea that pebbles made of $\mathrm{H}_{2} \mathrm{O}$ ice grains have a high sticking threshold velocity (Wada et al. 2009; Gundlach \& Blum 2015), but this is not the case if the grains are mantled by poorly sticky $\mathrm{CO}_{2}$ (Musiolik et al. 2016). Recent studies have found that this $\mathrm{CO}_{2}$-induced fragmentation can have important implications for the growth of pebble-accreting protoplanets (Johansen et al. 2019) and for the observational appearance of protoplanetary disks (Okuzumi \& Tazaki 2019). In a future work, we will study how the bouncing and fragmentation barriers affect the water delivery to rocky planets.

\subsection{Water fraction}

With the simulated pebble mass flux $\dot{M}_{\text {peb}}$, we calculate the growth rate of a planet due to icy pebble accretion $\left(\dot{M}_{\mathrm{pl}}\right)$. The filtering factor is defined by $f_{\mathrm{flt}}=\dot{M}_{\mathrm{pl}} / \dot{M}_{\mathrm{peb}}$. When the snowline passes each planetary orbit, we switch on the icy pebble accretion onto the planet. We assume that the 1:1 ratio of rocky to icy fraction of icy pebbles. We set the rocky planets with Venus, Earth, and Mars masses when the snowline passes 0.72, 1.0, and $1.52 \mathrm{au}$, respectively.

After the snowline passes a planetary orbit, icy pebble accretion starts. When the cumulative accreted mass by icy pebbles is $\Delta M_{\mathrm{pl}}$, the total ice mass in the planet is $(1 / 2) \Delta M_{\mathrm{pl}}$. The water fraction of the planet at the mass $M_{\mathrm{pl}}$ is given by

$f_{\text {water }}=\frac{(1 / 2) \Delta M_{\mathrm{pl}}}{M_{\mathrm{pl}}}=\frac{1}{2} \frac{\Delta M_{\mathrm{pl}}}{M_{\mathrm{pl}, 0}+\Delta M_{\mathrm{pl}}}$,

where $M_{\mathrm{pl}, 0}$ is the planetary mass at the snowline passage, $M_{\mathrm{pl}}=$ $M_{\mathrm{pl}, 0}+\Delta M_{\mathrm{pl}}$. If $\Delta M_{\mathrm{pl}}$ becomes much larger than $M_{\mathrm{pl}, 0}$, the water fraction saturates to $f_{\text {water }} \simeq 1 / 2$.

We repeat the simulations with different disk parameters $t_{\text {diff }}$, $\dot{M}_{\mathrm{g}, 0}, r_{\mathrm{d}, 0}$, and $\dot{M}_{\mathrm{pe}}$ to investigate how the water fraction of the final planets depends on these parameters and which values of the parameters produce the water fraction consistent with the terrestrial planets in the solar system. As we show in Sect. 4, the water fraction of final planets is insensitive to $M_{\mathrm{pl}, 0}$.

Figure 3 shows the time evolution of water fraction for the models with $t_{\text {diff }}=10^{6} \mathrm{yr}, \dot{M}_{\mathrm{g}, 0}=10^{-7} M_{\odot} \mathrm{yr}^{-1}$, and $\dot{M}_{\mathrm{pe}}=$ $10^{-9} M_{\odot} \mathrm{yr}^{-1}$. The left, middle, and right panels show the results of $r_{\mathrm{d}, 0}=30,100$, and $300 \mathrm{au}$, respectively. We first explain general evolution pattern of the water fraction. In all cases, the water fraction rapidly increases once the snowline passes the planetary orbit and the icy pebble accretion starts. It is saturated to its asymptotic value, even sufficiently before completion of disk gas depletion. From Eqs. (6) and (14),

$$
\begin{aligned}
r_{\text {snow,vis }} \simeq & 2.1\left(\frac{M_{*}}{M_{\odot}}\right)^{1 / 3} \\
& \times\left(\frac{r_{\mathrm{d}, 0}}{100 \mathrm{au}}\right)^{-2 / 9}\left(\frac{t_{\mathrm{diff}}}{10^{6} \mathrm{yr}}\right)^{2 / 9}\left(\frac{\dot{M}_{\mathrm{g}}}{10^{-7} M_{\odot} \mathrm{yr}^{-1}}\right)^{4 / 9} \mathrm{au} .
\end{aligned}
$$

At $t=0$ with $\dot{M}_{\mathrm{g}, 0}=10^{-7} M_{\odot} \mathrm{yr}^{-1}$, the snowline is located outside the orbit of Mars. As shown in Fig. 1, the snowline migrates inward and passes through the planetary orbits one after another as the disk accretion rate $\dot{M}_{\mathrm{g}}$ decreases with time as Eq. (10). The snowline passage always occurs in the order of Mars at $1.52 \mathrm{au}$, Earth at $1.0 \mathrm{au}$, and Venus at $0.72 \mathrm{au}$. Because the initial $r_{\text {snow,vis }}$ is closer to the planetary orbits for larger $r_{\mathrm{d}, 0}$, the snowline passage is earlier for larger $r_{\mathrm{d}, 0}$, as shown in Fig. 3. The water fraction is saturated when $\Sigma_{\mathrm{p}}$ and $\dot{M}_{\text {peb }}$ significantly decay. The rapid decay starts at $t \sim t_{\text {pff }}$ and Eq. (33) shows that $t_{\mathrm{pff}}$ is as short as approximately a few $\times 10^{5} \mathrm{yr}$ for $r_{\mathrm{d}, 0}=100 \mathrm{au}$. Even for $r_{\mathrm{d}, 0}=300 \mathrm{au}$, the decay starts at $t_{\mathrm{pff}} \simeq$ $10^{6} \mathrm{yr}$ before disk depletion. Therefore, the evolution of the water fraction evolution is mainly reduced by the consumption of icy dust grain reservoir rather than by the disk gas depletion.

Figure 3 shows a clear trend that the final water fraction is lower for smaller $r_{\mathrm{d}, 0}$. This trend is explained by a comparison between the snowline passage time $t_{\text {snow }}$ and $t_{\text {pff }}$, as follows. The water fraction due to pebble accretion rapidly increases until $\Sigma_{\mathrm{p}}$ decays by more than an order of magnitude, which corresponds to, say, $t>10 t_{\text {pff }}$ (Eq. (37)). If $t_{\text {snow }}>10 t_{\text {pff }}, f_{\text {water }}$ can be $\ll 1$. While $t_{\mathrm{pff}}$ is smaller for a smaller $r_{\mathrm{d}, 0}$ (Eq. (33)), $t_{\text {snow }}$ is larger (Eq. (40)). The latter implies that the disk is warmer for a smaller $r_{\mathrm{d}, 0}$. The viscous heating increases as the blanketing effect by optical depth $\left(\propto \Sigma_{\mathrm{g}}\right)$ increases. In Fig. 3, $\dot{M}_{\mathrm{g}, 0}$ and $t_{\text {diff }}$ are fixed. Smaller $r_{\mathrm{d}, 0}$ means smaller $\alpha$ (Eq. (13)) and larger $\Sigma_{\mathrm{g}}$ (Eq. (9)), resulting in a warmer disk. Thereby, the final water fraction is lower for smaller $r_{\mathrm{d}, 0}$.

We examine the condition of $t_{\text {snow }} / t_{\text {pff }}>10$ or $<10$ in more detail. In the case of $r_{\mathrm{d}, 0}=30 \mathrm{au}, t_{\mathrm{pff}} \simeq 4 \times 10^{4} \mathrm{yr}$ (Eq. (33)) and $t_{\text {snow }}$ is identified by the timing at which $f_{\text {water }}$ starts rapid increase, which is $\simeq 1 \times 10^{6}, 2.5 \times 10^{6}$, and $5 \times 10^{6} \mathrm{yr}$ for the Mars, Earth and Venus analogs, respectively (the left panel of Fig. 3). Because $t_{\text {snow }}>10 t_{\text {pff }}$ in this case, $f_{\text {water }} \sim 10^{-2}$ even for the outermost Mars analog. For the Earth and Venus analogs, $f_{\text {water }}$ is further smaller. In the case of $r_{\mathrm{d}, 0}=300 \mathrm{au}, t_{\mathrm{pff}} \simeq$ $1 \times 10^{6} \mathrm{yr}\left(\right.$ Eq. (33)) and $t_{\text {snow }} \simeq 1 \times 10^{5}, 1 \times 10^{6}$ and $2 \times 10^{6} \mathrm{yr}$ for the Mars, Earth, and Venus analogs, respectively (right panel of Fig. 3). Because $t_{\text {snow }}<10 t_{\text {pff }}, f_{\text {water }} \sim 1 / 2$ for all of Mars, Earth, and Venus. In the middle panel of Fig. 3, $r_{\mathrm{d}, 0}=100 \mathrm{au}$ and $t_{\mathrm{pff}} \simeq 2 \times 10^{5} \mathrm{yr}$ (Eq. (33)). In this case, $t_{\mathrm{snow}}<10 t_{\mathrm{pff}}$ and $f_{\text {water }} \ll 1$ for the Venus analog, while $t_{\text {snow }}>10 t_{\text {pff }}$ and $f_{\text {water }} \sim 1 / 2$ for the Mars analog.

The condition of $t_{\mathrm{snow}} / t_{\mathrm{pff}}>10$ or $<10$ also explains other results of the final water fraction of planets because this condition represents how much icy materials remain at the snowline passage. (In Sect. 5, we revisit this condition.) Figure 4 is the time evolution of water fraction of $t_{\text {diff }}=10^{6} \mathrm{yr}$ (left panel) and $t_{\mathrm{diff}}=3 \times 10^{6} \mathrm{yr}$ (right panel), respectively. The other disk parameters are the same. For both cases, $10 t_{\mathrm{pff}} \simeq 2 \times 10^{6} \mathrm{yr}$ (Eq. (33)). The snowline is already inside Mars' orbit from the beginning $(t=0)$ of the calculations, that is, $t_{\text {snow }}=0$, which results in $f_{\text {water }} \simeq 1 / 2$ for the Mars analog. The snowline passage time $t_{\text {snow }}$ is smaller than $10 t_{\text {pff }}$ only for the Venus analog in the case of $t_{\text {diff }}=10^{6} \mathrm{yr}$ (left panel), and for both the Earth 

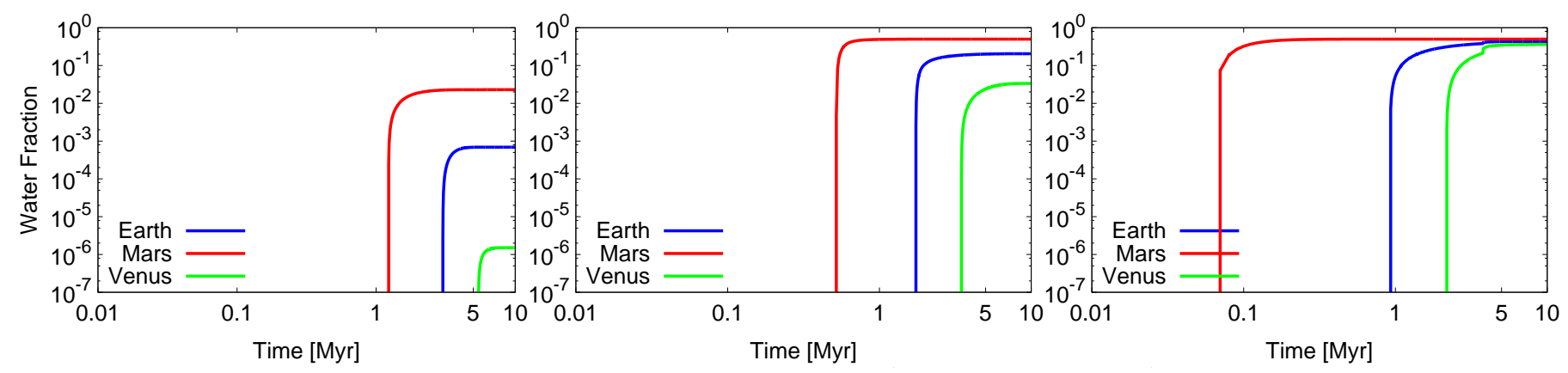

Fig. 3. Time evolution of water fraction for the models with $t_{\mathrm{diff}}=10^{6} \mathrm{yr}, \dot{M}_{\mathrm{g}, 0}=10^{-7} M_{\odot} \mathrm{yr}^{-1}$, and $\dot{M}_{\mathrm{pe}}=10^{-9} M_{\odot} \mathrm{yr}^{-1}$. Left, center, and right panel: results of $r_{\mathrm{d} .0}=30100$, and 300 au, respectively. The blue, red, and green lines represent the Earth, Mars and Venus analogs, respectively.

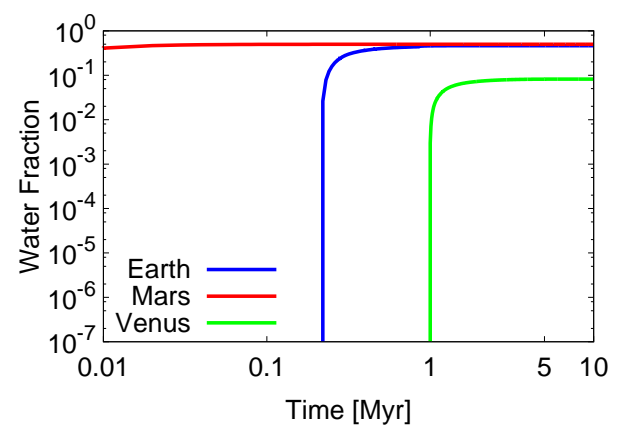

and Venus analogs in the case of $t_{\text {diff }}=3 \times 10^{6} \mathrm{yr}$ (right panel). This explains the results in Fig. 4.

Figure 5 shows the results of $\dot{M}_{\mathrm{g}, 0}=3 \times 10^{-8} M_{\odot} \mathrm{yr}^{-1}$ (left) and $10^{-7} M_{\odot} \mathrm{yr}^{-1}$ (right) with $r_{\mathrm{d}, 0}=100 \mathrm{au}, t_{\mathrm{diff}}=10^{6} \mathrm{yr}$, and $\dot{M}_{\mathrm{pe}}=10^{-9} M_{\odot} \mathrm{yr}^{-1}$. Again, $10 t_{\mathrm{pff}} \simeq 2 \times 10^{6} \mathrm{yr}$ for both cases. Smaller $\dot{M}_{\mathrm{g}, 0}$ with the fixed $t_{\text {diff }}=10^{6} \mathrm{yr}^{-1}$ means earlier passage of the snowline, resulting in a lower water fraction. Figure 6 shows the results of $\dot{M}_{\mathrm{pe}}=10^{-9} M_{\odot} \mathrm{yr}^{-1}$ (left) and $\dot{M}_{\mathrm{pe}}=$ $3 \times 10^{-9} M_{\odot} \mathrm{yr}^{-1}$ (middle) and $\dot{M}_{\mathrm{pe}}=10^{-8} M_{\odot} \mathrm{yr}^{-1}$. With larger $\dot{M}_{\text {pe }}$, the outer disk region is truncated at shorter radius. The disk truncation reduces the reservoir of icy materials, resulting in a lower water fraction, for the planets (the Earth and Venus analogs) with $t_{\text {snow }} \gtrsim 10 t_{\text {pff }} \simeq 2 \times 10^{6}$ yr.

In Sect. 4 , we derive a semi-analytical formula to predict the water fraction of planets after disk depletion. We show that the total mass $\left(M_{\mathrm{res}}\right)$ of the icy dust materials preserved in the disk determines the final water fraction because they eventually drift to the inner regions and pass the planetary orbits ${ }^{2}$. Since our gas disk model is analytical, we can analytically evaluate $t_{\text {snow }}$. We already know the analytical expression of $t_{\text {pff }}$ given by Eq. (36) is a good approximation. We also semi-analytically derive how the icy grain surface density evolves as Eq. (37). A synthesis of these results enables us to derive the semi-analytical formula for $f_{\text {water }}$.

Finally we point out that $f_{\text {water }}$ is higher in the order of Mars, Earth, and Venus analogs according to the snowline passage timing, except for the fully saturated cases where $f_{\text {water }} \sim 1 / 2$ for all the planets. We show that the final water fraction is insensitive to the embryo mass. The timing is the most important factor for $f_{\text {water }}$.

In the next section, we show that the derived semi-analytical expression of $f_{\text {water }}$ reproduces the numerical results. Using the

\footnotetext{
2 The grains at $r>300-500$ au would not undergo radial drift sufficiently, because their growth timescale is longer than $t_{\text {diff }}$. We consider disks with $r_{\mathrm{d}, 0} \leq 300$ au.
}

analytical expression, we clarify the dependence of the final water fraction on the disk parameters and pebble accretion parameters such as the initial planetary mass and Stokes number of accreting pebbles in Sect. 5. We also survey the disk parameter range that may reproduce $f_{\text {water }}$ comparable to that of the terrestrial planets in our solar system.

\section{Analytical formula for planetary water fraction}

The planetary water fraction is calculated by estimating the cumulative mass of accreted icy pebbles $\Delta M_{\mathrm{pl}}$ (Eq. (39)). We can simply estimate it as $\Delta M_{\mathrm{pl}} \simeq f_{\mathrm{ftt}}\left(M_{\mathrm{pl}}\right) \times M_{\text {res }}$, where $M_{\text {res }}$ is the total icy dust mass preserved in outer disk regions at the snowline passage because the pebble flux integrated from the snowline passage time $t_{\text {snow }}$ to infinity (effectively, to $t_{\text {diff }}$ ) must be similar to $M_{\text {res }}$.

Equation (39) is approximated to be

$$
\begin{aligned}
f_{\text {water }} & \simeq \frac{1}{2} \frac{f_{\mathrm{flt}}\left(M_{\mathrm{pl}}\right)}{M_{\mathrm{pl}, 0}+f_{\mathrm{flt}}\left(M_{\mathrm{pl}}\right) M_{\mathrm{res}}} M_{\mathrm{res}} \\
& \simeq \begin{cases}\frac{1}{2} \frac{f_{\mathrm{flt}}\left(M_{\mathrm{pl}, 0}\right)}{M_{\mathrm{pl}, 0}} M_{\mathrm{res}} & {\left[\Delta M_{\mathrm{pl}} \ll M_{\mathrm{pl}, 0}\right]} \\
\frac{1}{2} & {\left[\Delta M_{\mathrm{pl}} \gg M_{\mathrm{pl}, 0}\right],}\end{cases}
\end{aligned}
$$

where we use $f_{\mathrm{flt}}\left(M_{\mathrm{pl}}\right) \simeq f_{\mathrm{ftt}}\left(M_{\mathrm{pl}, 0}\right)$ for $\Delta M_{\mathrm{pl}} \ll M_{\mathrm{pl}, 0}$ in the upper line. For the range of the disk parameters we use in numerical simulations, the accretion is mostly in the 3D regime (Eq. (28)) and $f_{\mathrm{flt}} \propto M_{\mathrm{pl}}$. In that case, Eq. (41) shows that $f_{\text {water }}$ is independent of $M_{\mathrm{pl}}$ both in the cases of $\Delta M_{\mathrm{pl}}<M_{\mathrm{pl}, 0}$ and $\Delta M_{\mathrm{pl}}>M_{\mathrm{pl}, 0}$, while it depends on the disk parameters. Even if the transition to $2 \mathrm{D}$ accretion occurs at a smaller planet mass (which happens for smaller values of $\alpha$ ), the mass dependence of $f_{\text {water }}$ is still weak: it is proportional to $M_{\mathrm{pl}}^{-1 / 3}$ for $\Delta M_{\mathrm{pl}}<M_{\mathrm{pl}, 0}$ and independent of $M_{\mathrm{pl}}$ otherwise. We can combine the two limits in Eq. (41) by a 

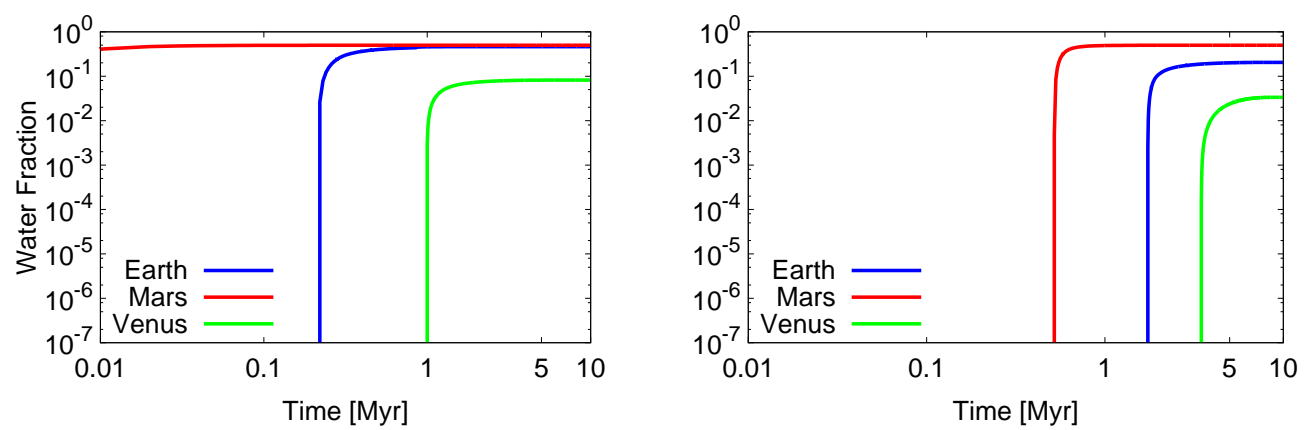

Fig. 5. Time evolution of water fraction for the models with $r_{\mathrm{d} .0}=100 \mathrm{au}$, $t_{\text {diff }}=10^{6} \mathrm{yr}$ and $\dot{M}_{\mathrm{pe}}=10^{-9} M_{\odot} \mathrm{yr}^{-1}$. Left and right panels: results of $\dot{M}_{\mathrm{g}, 0}=3 \times 10^{-8} \quad$ and $\quad 10^{-7} M_{\odot} \mathrm{yr}^{-1}$, respectively.
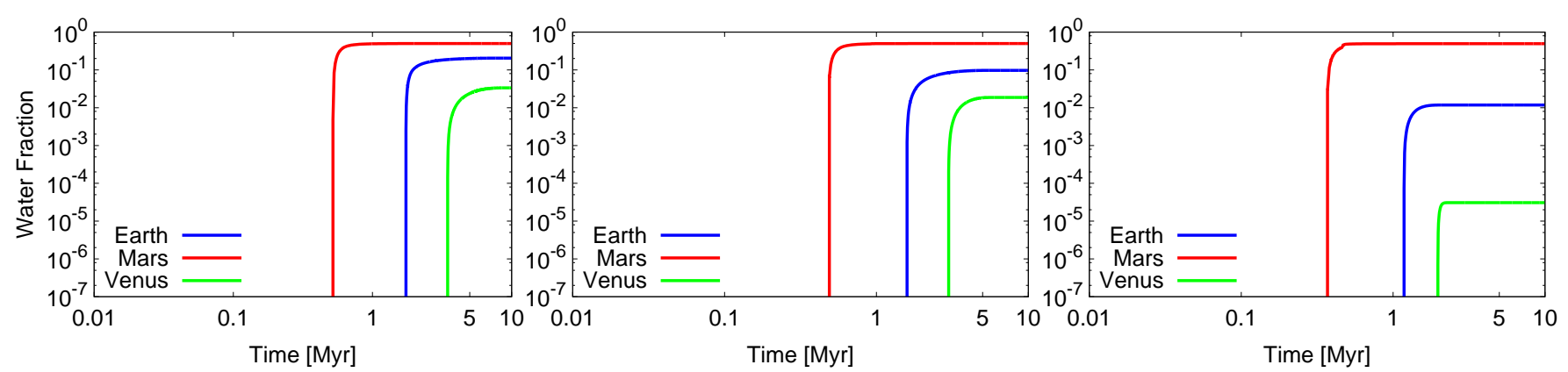

Fig. 6. Time evolution of water fraction for the models with $r_{\mathrm{d} .0}=100 \mathrm{au}, t_{\mathrm{diff}}=10^{6} \mathrm{yr}$ and $\dot{M}_{\mathrm{ini}}=10^{-7} M_{\odot} \mathrm{yr}^{-1}$. Left, center, and right panels: results of $\dot{M}_{\mathrm{pe}}=10^{-9}, 3 \times 10^{-9}$, and $10^{-8} M_{\odot} \mathrm{yr}^{-1}$, respectively.

simple formula as

$f_{\mathrm{water}} \simeq \frac{1}{2} \frac{f_{\mathrm{flt}}\left(M_{\mathrm{pl}, 0}\right)}{M_{\mathrm{pl}, 0}+f_{\mathrm{flt}}\left(M_{\mathrm{pl}, 0}\right) M_{\mathrm{res}}} M_{\mathrm{res}}$.

In Fig. $7, f_{\text {water,sim obtained by our simulations is compared }}$ with Eq. (42) with the simulated $M_{\text {res }}$. This figure shows that Eq. (42) reproduces the numerical results very well. Both the numerical results and Eq. (42) show $f_{\text {water }} \propto M_{\text {res }}$ for $f_{\text {water }} \ll$ $1 / 2$. The icy dust mass $M_{\text {res }}$ depends sensitively on the disk parameters, $\dot{M}_{\mathrm{g}, 0}, \dot{M}_{\mathrm{pe}}, t_{\mathrm{diff}}$, and $r_{\mathrm{d}, 0}$. On the other hand, $f_{\mathrm{flt}}$ in $2 \mathrm{D}$ is independent of these disk parameters, while that in $3 \mathrm{D}$ depends only weakly $\left(\propto \sqrt{r_{\mathrm{d}, 0} / t_{\mathrm{diff}}}\right)$. In the case of $\Delta M_{\mathrm{pl}} \ll$ $M_{\mathrm{pl}, 0}, f_{\text {water }} \simeq\left(f_{\mathrm{flt}} / M_{\mathrm{pl}, 0}\right) M_{\text {res }}$. As we discussed, $f_{\mathrm{flt}} / M_{\mathrm{pl}, 0}$ is almost constant, so that $f_{\text {water }}$ should be almost proportional to $M_{\text {res }}$ for $\Delta M_{\mathrm{pl}} \ll M_{\mathrm{pl}, 0}$.

Because the analytical solution given by Eq. (42) reproduces the numerical results, we next derive an analytical formula for the icy dust mass $M_{\text {res }}$ at the snowline passage as follows. From Eq. (6), the snowline passes the planet orbit at $a_{\mathrm{pl}}$ when

$$
\begin{aligned}
\dot{M}_{\mathrm{g}} & \simeq \dot{M}_{\mathrm{g}, \text { snow }} \\
& =2 \times 10^{-8}\left(\frac{a_{\mathrm{pl}}}{1 \mathrm{au}}\right)^{9 / 4}\left(\frac{M_{*}}{M_{\odot}}\right)^{-3 / 4}\left(\frac{\alpha}{10^{-2}}\right)^{1 / 2} M_{\odot} \mathrm{yr}^{-1} .
\end{aligned}
$$

Using Eq. (14),

$$
\begin{aligned}
\dot{M}_{g, \text { snow }}= & 2 \times 10^{-8}\left(\frac{M_{*}}{M_{\odot}}\right)^{-3 / 4} \\
& \times\left(\frac{a_{\mathrm{pl}}}{1 \mathrm{au}}\right)^{9 / 4}\left(\frac{t_{\mathrm{diff}}}{10^{6} \mathrm{yr}}\right)^{-1 / 2}\left(\frac{r_{\mathrm{d}, 0}}{100 \mathrm{au}}\right)^{1 / 2} M_{\odot} \mathrm{yr}^{-1} .
\end{aligned}
$$

From Eq. (10), the snowline passage time is

$\tilde{t}_{\text {snow }} \simeq\left(\frac{\dot{M}_{\mathrm{g}, 0}}{\dot{M}_{g, \text { snow }}+\dot{M}_{\mathrm{pe}}}\right)^{2 / 3} ; t_{\text {snow }} \simeq\left[\left(\frac{\dot{M}_{\mathrm{g}, 0}}{\dot{M}_{g, \text { snow }}+\dot{M}_{\mathrm{pe}}}\right)^{2 / 3}-1\right] t_{\mathrm{diff}}$.

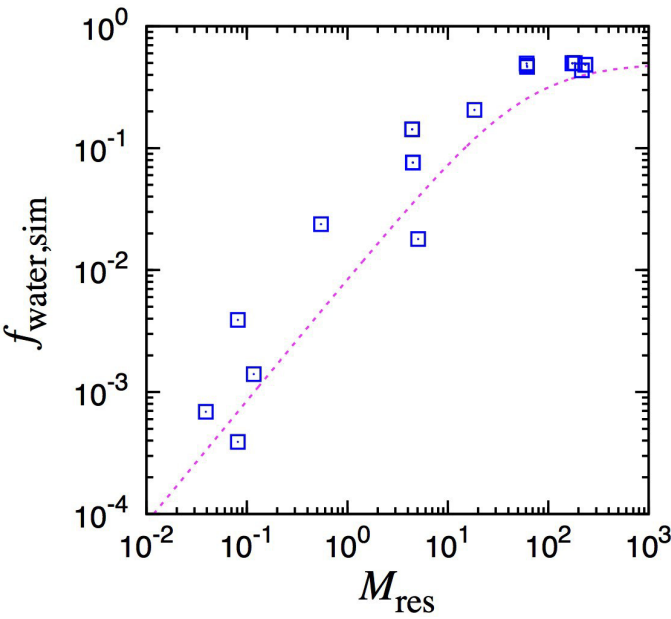

Fig. 7. Water fraction $f_{\text {water,sim }}$ and icy dust mass preserved in the outer disk at the snowline passage $M_{\text {res }}$ obtained by our simulations. The blue squares show the simulation results for the Earth analogs with different values of $\dot{M}_{\mathrm{g}, 0}, \dot{M}_{\mathrm{pe}}, t_{\mathrm{diff}}$, and $r_{\mathrm{d}, 0}$ (Sect. 2.4). The dotted curve represents the analytical solution given by Eq. (42).

From Eq. (12), the remaining gas disk mass at the snowline passage is given by

$M_{\mathrm{g}, \text { snow }} \simeq 2 \tilde{t}_{\text {snow }} t_{\text {diff }}\left(\dot{M}_{\mathrm{g}, 0} \tilde{t}_{\text {snow }}^{-3 / 2}-\dot{M}_{\mathrm{pe}}\right)$.

Because $M_{\text {res }} \sim Z M_{\mathrm{g}, \text { snow }}$, we obtain

$M_{\text {res }} \sim 2 Z_{0}\left(1+\frac{t_{\text {snow }}}{t_{\text {pff }}}\right)^{-\gamma} \tilde{t}_{\text {snow }} t_{\text {diff }}\left(\dot{M}_{\mathrm{g}, 0} \tilde{t}_{\text {snow }}^{-3 / 2}-\dot{M}_{\mathrm{pe}}\right)$,

where we approximated $Z$ as $Z \sim\left(1+t / t_{\mathrm{pff}}\right)^{-\gamma} Z_{0}$ from Eq. (37), $t_{\mathrm{pff}} \sim 2 \times 10^{5}(r / 100 \mathrm{au})^{3 / 2}\left(M_{*} / M_{\odot}\right)^{-1 / 2} \mathrm{yr}^{-1}$ (Eq. (36)), and $\gamma=$ $1+0.15\left(300 \mathrm{au} / r_{\mathrm{d}, 0}\right)($ Eq. (38)). Substituting the filtering factor $f_{\text {flt }}$ given by Eq. (29) with $\mathrm{St}=0.1$ and $M_{\text {res }}$ estimated above into Eq. (42), we can analytically estimate the water fraction 


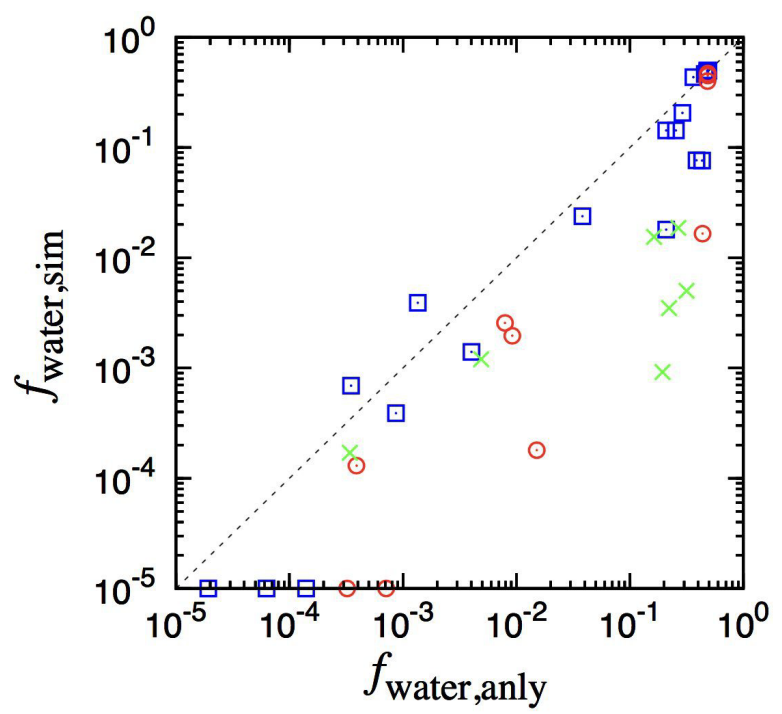

Fig. 8. Comparison of the water fraction obtained by the numerical simulations $\left(f_{\text {water,sim }}\right)$ with the analytical estimate $\left(f_{\text {water,anly }}\right)$. The blue squares, red circles, and green cross represent the results for the Earth, Mars, and Venus analogs, respectively. If $f_{\text {water,sim }}$ obtained by the numerical simulation is smaller than $10^{-5}$, we put $f_{\text {water,sim }}=10^{-5}$ because the numerical results would include a numerical uncertainty for such small values of $f_{\text {water,sim }}$.

from the disk parameters, $\dot{M}_{\mathrm{g}, 0}, \dot{M}_{\mathrm{pe}}, t_{\mathrm{diff}}$, and $r_{\mathrm{d}, 0}$ as (Eqs. (29) and (39))

$f_{\text {water }} \simeq \frac{1}{2}\left(1+\frac{M_{\mathrm{pl}, 0}}{f_{\mathrm{flt}}\left(M_{\mathrm{pl}, 0}\right) M_{\mathrm{res}}}\right)^{-1}$,

where $M_{\text {res }}$ is given by Eq. $(47), f_{\mathrm{flt}}=\min \left(f_{\mathrm{flt}, 3 \mathrm{D}}, f_{\mathrm{flt}, 2 \mathrm{D}}\right)$, and

$$
\begin{aligned}
f_{\mathrm{flt}, 3 \mathrm{D}} \simeq & 0.017\left(\frac{M_{*}}{M_{\odot}}\right)^{-1}\left(\frac{\alpha}{10^{-2}}\right)^{-1 / 2}\left(\frac{h_{\mathrm{g}} / r}{0.02}\right)^{-1}\left(\frac{S t}{0.1}\right)^{1 / 2} \\
& \times\left(\frac{M_{\mathrm{pl}, 0}}{0.1 M_{\oplus}}\right)\left(\frac{r}{1 \mathrm{au}}\right)^{-1 / 2}, \\
f_{\mathrm{flt}, 2 \mathrm{D}} \simeq & 0.040\left(\frac{M_{*}}{M_{\odot}}\right)^{-2 / 3}\left(\frac{S t}{0.1}\right)^{-1 / 3}\left(\frac{M_{\mathrm{pl}, 0}}{0.1 M_{\oplus}}\right)^{2 / 3}\left(\frac{r}{1 \mathrm{au}}\right)^{-1 / 2}
\end{aligned}
$$

Except for the value of $\gamma$, which was fitted by the numerical results, the other derivations are analytical. We note that $f_{\text {water }} \propto$ $M_{\text {res }} \propto Z_{0}$. Around metal-rich stars, $f_{\text {water }}$ would be larger.

In Fig. 8, we compare the analytically estimated water fraction $f_{\text {water,anly }}$ with the numerically simulated $f_{\text {water,sim. In }}$ addition to the Earth analogs $\left(M_{\mathrm{pl}, 0}=1 M_{\oplus}\right)$ at $1 \mathrm{au}$, we also plot the results for the Mars analogs $\left(M_{\mathrm{pl}, 0}=0.11 M_{\oplus}\right)$ at $1.52 \mathrm{au}$ and the Venus analogs $\left(M_{\mathrm{pl}, 0}=0.82 M_{\oplus}\right)$ at $0.72 \mathrm{au}$. For the Earth and Mars analogs, $f_{\text {water,anly }}$ reproduces $f_{\text {water,sim }}$ within a factor of several, while the water fraction varies by several orders of magnitude, except for some runs for which the water fraction is overestimated by the analytical formula. The agreement both for the Earth and Mars analogs strongly suggests that the mass and semimajor axis dependences are also reproduced. In the case of Venus analogs, $f_{\text {water,anly }}$ is larger by a factor of a few to a few tens than $f_{\text {water,sim. }}$. When the Earth analog increases its mass by capture of pebbles, it captures a significant fraction of the pebble flux and the accretion of the Venus analogs that reside in inner region of the Earth analogs can be significantly decreased. This effect is included in the numerical simulation, while it is not taken into account in the analytical formula.

\section{Dependence of water fraction on disk parameters}

Using the analytical formula, we investigate how the water fraction $\left(f_{\text {water }}\right)$ is determined by the disk parameters. Figure 9 shows $f_{\text {water }}$ for a planet at $a_{\mathrm{pl}}=1.0 \mathrm{au}$ as a function of the disk parameters, $\dot{M}_{\mathrm{g}, 0}$ and $r_{\mathrm{d}, 0}$. The other parameters are $\dot{M}_{\mathrm{pe}}=10^{-9} M_{\odot} \mathrm{yr}^{-1}$ and $t_{\mathrm{diff}}=3 \times 10^{6} \mathrm{yr}^{-1}$. The panels a show the dependence on $M_{\mathrm{pl}, 0}$ for $S t=0.1$. The panels b show the dependence on $S t$ for $M_{\mathrm{pl}, 0}=1 M_{\oplus}$. The planets formed with the parameters in the red region are very dry, $f_{\text {water }} \lesssim 10^{-4}$. Those in the green region have a modest amount of water, $f_{\text {water }} \sim 0.1$, and those in the blue region are icy planets, $f_{\text {water }} \simeq 1 / 2$. The yellow and orange regions represent $f_{\text {water }} \sim 10^{-4}-10^{-2}$, which corresponds to the water fraction of the current Earth and that estimated for ancient Mars.

We find that $f_{\text {water }}$ is the most sensitive to $\dot{M}_{\mathrm{g}, 0}$ and $r_{\mathrm{d}, 0}$. The common features in the contours in Fig. 9 are that (i) $f_{\text {water }}$ is lower for smaller $r_{\mathrm{d}, 0}$ and larger $\dot{M}_{\mathrm{g}, 0}$, and (ii) $f_{\text {water }} \sim 10^{-4}-10^{-2}$ is realized at $r_{\mathrm{d}, 0} \sim 30-50$ au and $\dot{M}_{\mathrm{g}, 0} \gtrsim 10^{-8} M_{\odot} \mathrm{yr}^{-1}$. This is consistent with the conclusion by Sato et al. (2016) that such $f_{\text {water }}$ can be realized for compact disks with sizes $<100$ au and late snowline passage $(>2-4 \mathrm{Myr})$. We show that the disk parameters for $f_{\text {water }} \sim 10^{-4}-10^{-2}$ is not in a very narrow window.

As we showed in Sect. $4, f_{\text {water }}$ is regulated by $M_{\text {res }}$ and $M_{\text {res }}$ is sensitive to $\dot{M}_{\mathrm{g}, 0}$ and $r_{\mathrm{d}, 0}$, especially through the parameter $t_{\text {snow }} / t_{\text {pff }}($ Eq. (47)). Because pebble accretion is fast to realize $f_{\text {water }} \sim 10^{-4}-10^{-2}, M_{\text {res }}$ has to have significantly decayed until $t \sim t_{\text {snow }}$. For small $r_{\mathrm{d}, 0}, t_{\text {pff }}$ is small (Eq. (36)), while $t_{\text {snow }}$ is large (Eq. (45)) due to small $\dot{M}_{\mathrm{g} \text {,snow }}$ (Eq. (44)). For large $\dot{M}_{\mathrm{g}, 0}, t_{\text {snow }}$ is small (Eq. (44)) while $t_{\text {pff }}$ is the same (Eq. (36)). As a result, $\Sigma_{\mathrm{p}}$ decays more quickly (Eq. (37)) for small $r_{\mathrm{d}, 0}$ and large $\dot{M}_{\mathrm{g}, 0}$.

Figure 9 shows the dependences of $f_{\text {water }}$ on the pebble accretion parameters: the initial planetary mass $\left(M_{\mathrm{pl}, 0}\right)$ and the Stokes number (St) of pebbles that accrete onto the planet.

In the numerical simulations, the Stokes number of pebbles that accrete onto the planet is calculated by growth and radial drift of pebbles in an evolving disk. According to the simulations, the Stokes number of radially drifting particles is $\sim 0.1$ at early times (Eq. (34)), but decreases with time as the dust and gas disks evolve. As Eq. (37) shows, $\Sigma_{\mathrm{p}}$ decays more rapidly than $\Sigma_{\mathrm{g}}$ for $t>t_{\mathrm{pff}}$. Accordingly, $Z$ decreases and the equilibrium Stokes number of migrating pebbles become smaller (Eq. (34); also see Sato et al. 2016). As we pointed out in Sect. 3.1, if a fragmentation/rebound barrier limits the icy pebble growth, $S t$ also becomes small. With this reasoning, we also showed plots with $S t=0.01$ and 0.001 in Fig. 9.

Figure 9 shows that $f_{\text {water }}$ is almost independent of $M_{\mathrm{pl}, 0}$ and St. The weak dependence on $M_{\mathrm{pl}, 0}$ is explained by the following arguments. The water fraction $f_{\text {water }}$ is $\sim\left(f_{\mathrm{ft}} / M_{\mathrm{pl}, 0}\right) M_{\text {res }}$ in the case of $\Delta M_{\mathrm{pl}} \ll M_{\mathrm{pl}, 0}$. The dust mass $M_{\mathrm{res}}$ is independent of $M_{\mathrm{pl}}$. As already explained in Sect. 4 , the factor $f_{\mathrm{flt}} / M_{\mathrm{pl}, 0}$ is independent of $M_{\mathrm{pl}, 0}$ in 3D accretion and only weakly depends on $M_{\mathrm{pl}, 0}$ in $2 \mathrm{D}$ accretion $\left(\propto M_{\mathrm{pl}, 0}^{-1 / 3}\right)$.

The weak dependence of $f_{\text {water }}$ on Stokes number shown in Fig. 9 is the result of the assumption that $M_{\text {res }}$ is independent of $S t$; it depends on $S t$ only weakly (Eq. (29)) through $f_{\text {flt }}$. The parameter $\gamma$ could be dependent on $S t$ because the sculpture rate of $\Sigma_{\mathrm{p}}$ at $r=r_{\mathrm{pff}}$ may depend on St. Figure 10 shows the dependence on $\gamma_{2}$, assuming that the functional form of $\gamma=1+\gamma_{2}\left(300 \mathrm{au} / r_{\mathrm{d}, 0}\right)$ still holds. Because the sculpture rate may be lower for smaller values of $S t$, we tested the cases of 

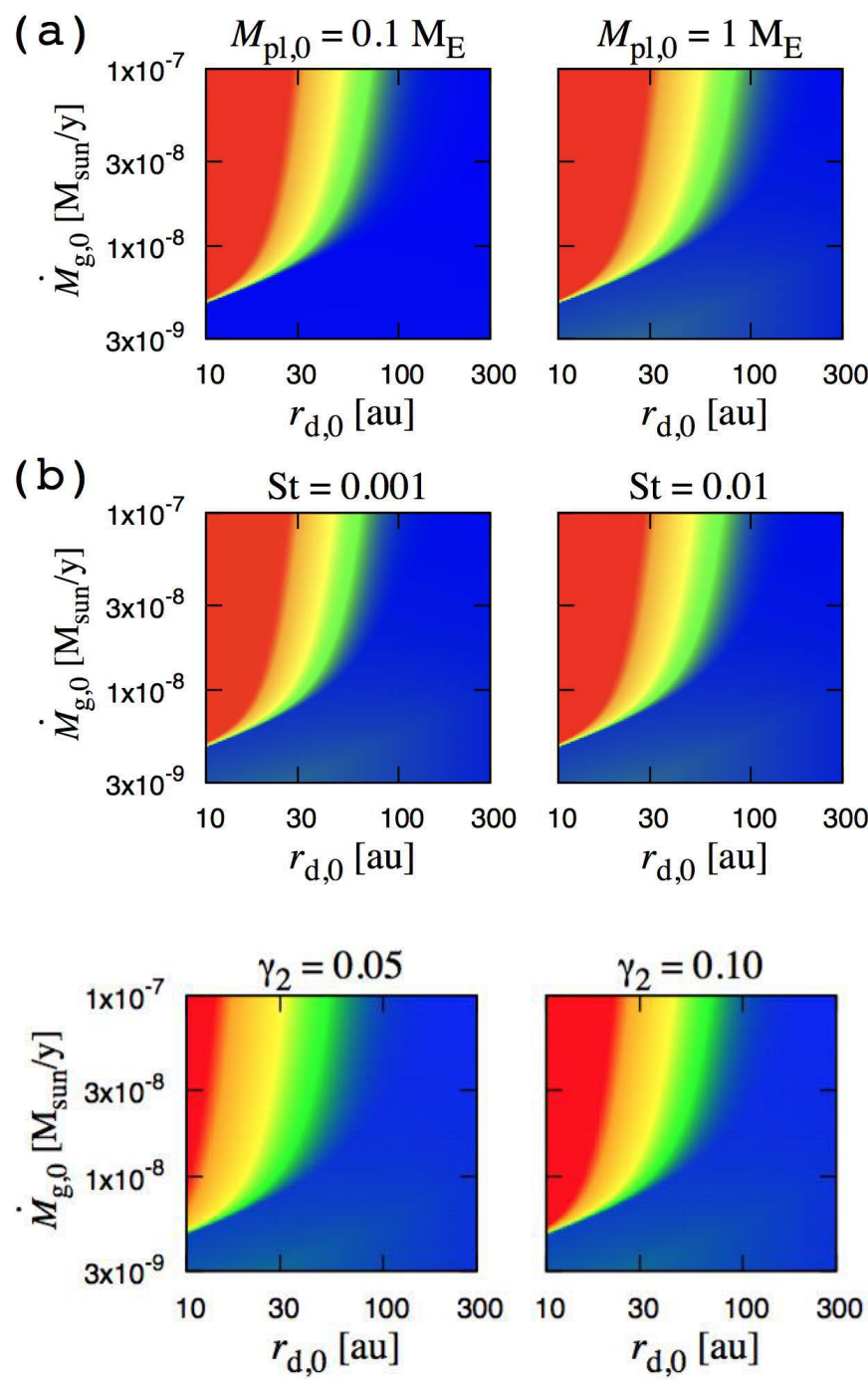

$\gamma_{2}=0.05$ and 0.1 , in addition to the nominal case of $\gamma_{2}=0.15$. The result is insensitive to $\gamma_{2}$ for $\gamma_{2} \gtrsim 0.1$, while the sculpture rate is lower and accordingly the $f_{\text {water }}$ is relatively higher for $\gamma_{2} \sim 0.05$. The detailed functional form of $\gamma$ is left for future works.

Figure 11 shows the dependence on the other disk parameters, $t_{\text {diff }}$ and $\dot{M}_{\text {pe }}$. For larger $t_{\text {diff }}$, the evolution of the snowline is slower and the snowline passage is later (Eq. (45)). Before the passage, the icy dust disk has been more sculpted. As a result, $M_{\text {res }}$ is smaller (Eq. (47)) because $t_{\text {snow }} \propto t_{\text {diff }}$ and $\gamma \geq 1$. For smaller $\dot{M}_{\text {pe }}$, the disk is hotter and the snowline passage is later ( $t_{\text {snow }}$ is larger), resulting in smaller $M_{\text {res }}$. If $M_{\text {res }}$ is smaller, $f_{\text {water }}$ is smaller. The water fraction corresponding to the current Earth is $10^{-4}-10^{-2}$ (the yellow and orange colored regions) are only slightly shifted to larger $r_{\mathrm{d}, 0}$ and lower $\dot{M}_{\mathrm{g}, 0}$ for smaller $t_{\text {diff }}$ and larger $\dot{M}_{\text {pe }}$, because $M_{\text {res }}$ is smaller for these parameters (Eq. (47)). We note that the parameter region with $\dot{M}_{\mathrm{g}, 0}<\dot{M}_{\mathrm{pe}}$ is empty in the right panel of Fig. 11b because the disks do not exist under that condition.

In Fig. 12, the analytically estimated $f_{\text {water }}$ is plotted for $a_{\mathrm{pl}}=$ 0.72, 1.00 and 1.52 au (the Venus, Earth, and Mars analogs, respectively). In the outer region, $f_{\text {water }}$ is generally larger due to early snowline passage (smaller $t_{\text {snow }}$ ). In other words, $\dot{M}_{\mathrm{g} \text {,snow }}$ is larger for larger $a_{\mathrm{pl}}$. As mentioned in Sect. 4, the Venus analogs may have further lower $f_{\text {water }}$, if we take into account the decrease
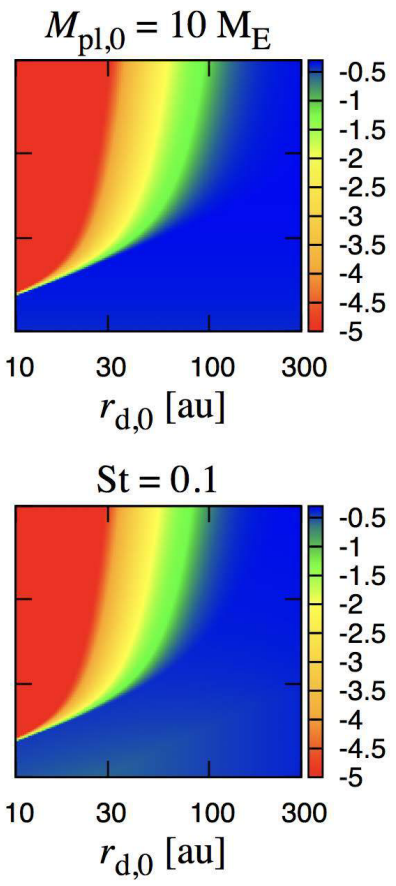

Fig. 9. Analytical estimate of $f_{\text {water }}$ at $1 \mathrm{au}$ as a function of the initial disk radius $\left(r_{\mathrm{d}, 0}\right)$ and the initial disk accretion rate $\left(\dot{M}_{\mathrm{g}, 0}\right)$. Panels $a$ : dependence on the planet mass $\left(M_{\mathrm{pl}, 0}\right)$. Panels $b$ : Stokes number $(S t)$ of pebbles. The other parameters are $\dot{M}_{\mathrm{pe}}=10^{-9} M_{\odot} \mathrm{yr}^{-1}$ and $t_{\text {diff }}=3 \times 10^{6} \mathrm{yr}$. In the panels a, we use $S t=0.1$. In the panels b, $M_{\mathrm{pl}, 0}=1 M_{\oplus}$. The middle panels in a and $\mathrm{b}$ are identical. The color scales are $\log _{10} f_{\text {water }}$.

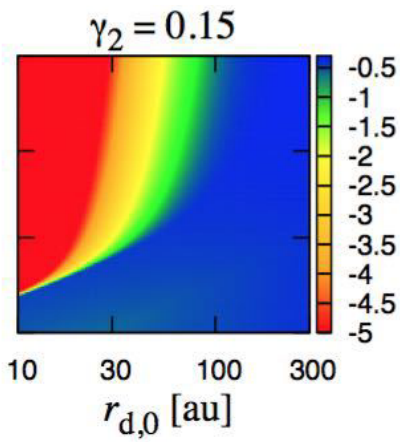

Fig. 10. Analytical estimate of $f_{\text {water }}$ at $1 \mathrm{au}$ as a function of the initial disk radius $\left(r_{\mathrm{d}, 0}\right)$ and the initial disk accretion rate $\left(\dot{M}_{\mathrm{g}, 0}\right)$, where $\gamma_{2}=0.05,0.10$, and 0.15 . The other parameters are $S t=0.1$, $M_{\mathrm{pl}, 0}=1 M_{\oplus}, \quad \dot{M}_{\mathrm{pe}}=10^{-9} M_{\odot} \mathrm{yr}^{-1^{-1}}$, and $t_{\text {diff }}=3 \times 10^{6} \mathrm{yr}$. The color scales are $\log _{10} f_{\text {water }}$.

in the pebble flux due to accretion by the Earth analog. As shown in Eq. (7), the snowline cannot reach the region inside 0.53 au in our disk model. The planets there are completely dry. Although the dependence on the orbital radius exists, in the case of $r_{\mathrm{d}, 0} \sim 30-50$ au and $\dot{M}_{\mathrm{g}, 0} \gtrsim 2 \times 10^{-8} M_{\odot} \mathrm{yr}^{-1}, f_{\text {water }} \sim 10^{-4}-10^{-2}$ for both the Earth and Mars analogs.

In Sect. 3, we pointed out that the simple condition of $t_{\text {snow }} / t_{\text {pff }}<10$ or $>10$ discriminates between the water-rich case $\left(f_{\text {water }} \sim 1 / 2\right)$ and the water-poor case. The pebble formation timescale $t_{\mathrm{pff}}$ is given by Eq. (36) and the snowline passage time $t_{\text {snow }}$ is given by Eq. (45). Both $t_{\text {pff }}$ and $t_{\text {snow }}$ are independent of $M_{\mathrm{pl}}$ and St, which is consistent with Fig. 9. The condition of $t_{\text {snow }}=10 t_{\mathrm{pff}}$ is shown on the $r_{\mathrm{d}, 0}-\dot{M}_{\mathrm{g}, 0}$ plane in Fig. 13 . We show the dependences on $t_{\mathrm{diff}}, \dot{M}_{\mathrm{pe}}$, and $a_{\mathrm{pl}}$. A comparison of this figure with Figs. 11 and 12 show that the simple condition approximately reproduces the more detailed evaluation except for $r_{\mathrm{d}, 0}>100 \mathrm{au}$. As shown in Fig. 1, for $r_{\mathrm{d}, 0}>100 \mathrm{au}$, the disk radius expands with the pebble formation front radius $r_{\mathrm{pff}}$, so that the sculpture of icy dust reservoir is delayed, compared to the estimation of $t_{\mathrm{pff}}$ at $r_{\mathrm{d}, 0}$. The calculation of $M_{\mathrm{res}}$ does not have this problem.

As we have shown, $f_{\text {water }}$ is the most sensitive to $\dot{M}_{\mathrm{g}, 0}$ and $r_{\mathrm{d}, 0}$ and almost independent of the other parameters of disks and pebble accretion. A robust result would be that the water fraction inferred for the present Earth and ancient Mars, 
(a)

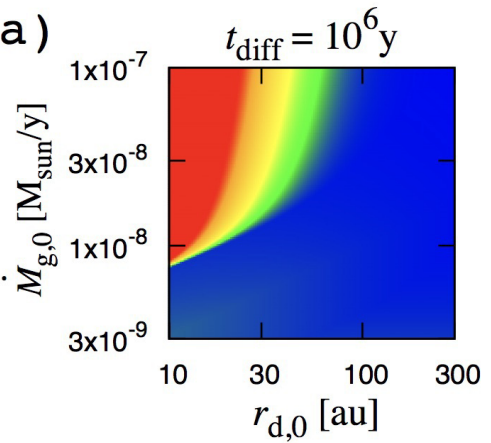

(b)
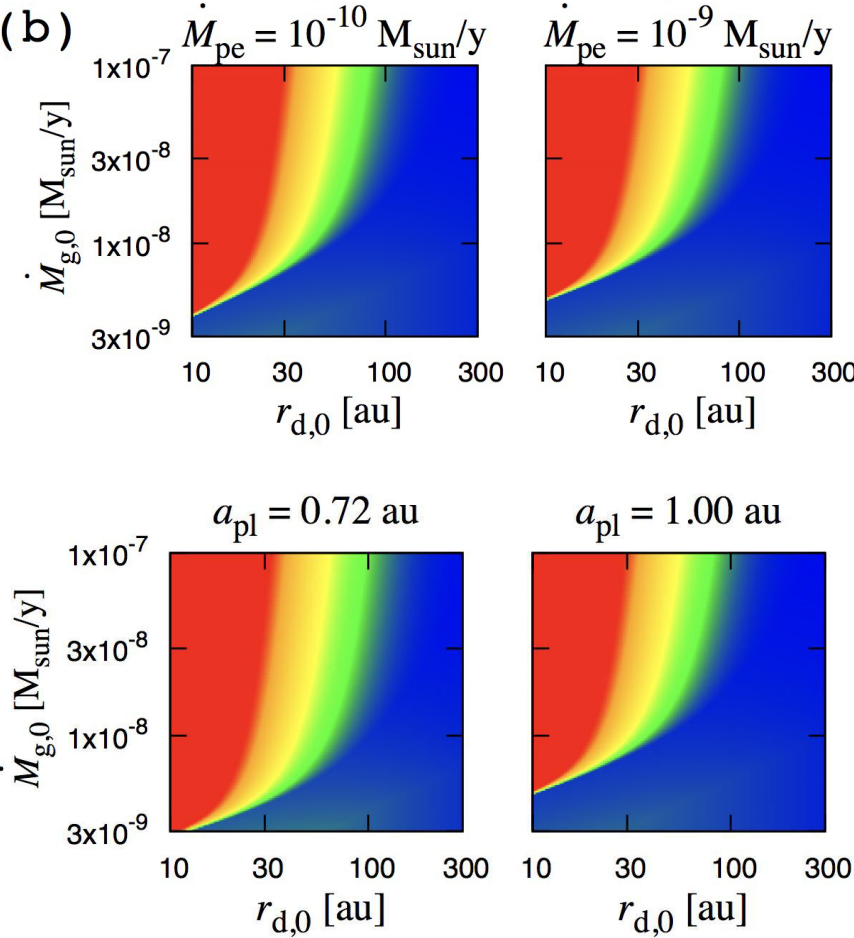

$f_{\text {water }} \sim 10^{-4}-10^{-2}$, is realized at $r_{\mathrm{d}, 0} \sim 30-50$ au and $\dot{M}_{\mathrm{g}, 0} \gtrsim$ $2 \times 10^{-8} M_{\odot} \mathrm{yr}^{-1}$, which may correspond to median disks of classical $\mathrm{T}$ Tauri stars or slightly compact and massive disks.

\section{Discussion}

In this section, we comment on the effect of pebble isolation mass, which we did not include in our simulations. A planet with relatively large mass $\left(M_{\mathrm{pl}}\right)$ can make a dip in the gas disk along the planetary orbit to prevent the pebbles from passing the orbit. The threshold mass is called "pebble isolation mass" $\left(M_{\text {peb,iso }}\right.$; Lambrechts et al. 2014; Bitsch et al. 2018; Ataiee et al. 2018). When the planetary mass reaches the isolation mass, pebble accretion onto the planet with $M_{\mathrm{pl}}>M_{\text {peb,iso }}$ and other planets inside the planetary orbit is truncated and the increases in their water fraction are stalled.

Bitsch et al. (2018) derived a detailed expression of the pebble isolation mass,

$M_{\text {peb,iso }} \simeq 25\left(\frac{h / r}{0.05}\right)^{3}\left[0.34\left(\frac{3}{\log _{10}(\alpha)}\right)^{4}+0.66\right] M_{\oplus}$.

For example, $M_{\text {peb,iso }} \simeq 4.1 M_{\oplus}$ for $t_{\text {diff }}=3 \times 10^{6} \mathrm{yr}, \dot{M}_{\mathrm{g}, 0}=$ $10^{-8} M_{\odot} \mathrm{yr}^{-1}, r_{\mathrm{d}, 0}$ au, $h / r \simeq 0.0246$ (at 1 au), and $\alpha \simeq 3 \times$ $10^{-3}$. If $M_{\mathrm{pl}, 0} \gtrsim$ several $M_{\oplus}$, the effect of pebble isolation mass is not negligible. Figure 14 shows the water fraction for

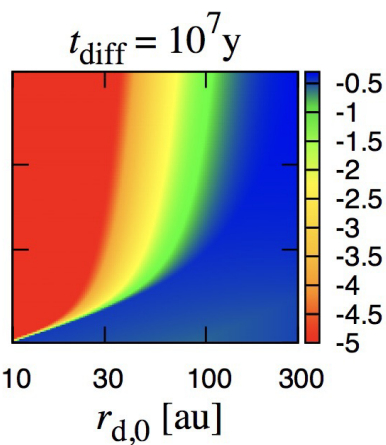

Fig. 11. Analytical estimate of $f_{\text {water }}$ at $1 \mathrm{au}$ as a function of $r_{\mathrm{d}, 0}$ and $\dot{M}_{\mathrm{g}, 0}$. Panels $a$ : The dependence on the disk diffusion timescale $t_{\text {diff. Panels }} b$ : dependence on the photoevaporation rate $\left(\dot{M}_{\mathrm{pe}}\right)$. The other parameters are $S t=0.1$ and $M_{\mathrm{pl}, 0}=1 M_{\oplus}$. In the panels a, we used $\dot{M}_{\mathrm{pe}}=10^{-9} M_{\odot} \mathrm{yr}^{-1}$, and $t_{\text {diff }}=3 \times 10^{6} \mathrm{yr}$ in the panels $\mathrm{b}$. The middle panels of panels a and the right panel of panels $b$ are identical. In the right panel of panel $\mathrm{b}$, the region with $\dot{M}_{\mathrm{g}, 0}<\dot{M}_{\mathrm{pe}}$ is empty because the disks do not exist under that condition. The color scales are $\log _{10} f_{\text {water }}$.

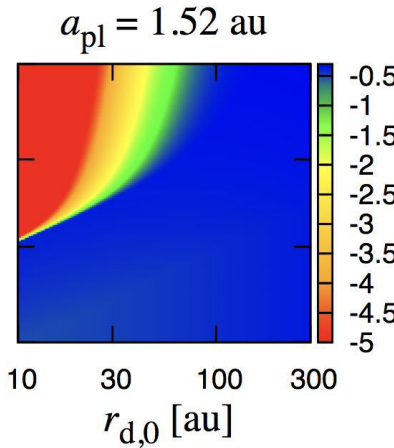

Fig. 12. Analytical estimate of $f_{\text {wate }}$ as a function of $r_{\mathrm{d}, 0}$ and $\dot{M}_{\mathrm{g}, 0}$, at 0.72 , 1.00 , and $1.52 \mathrm{au}$. The other parameters are $S t=0.1, M_{\mathrm{pl}, 0}=1 M_{\oplus}, \dot{M}_{\mathrm{pe}}=$ $10^{-9} M_{\odot} \mathrm{yr}^{-1}$, and $t_{\mathrm{diff}}=3 \times 10^{6} \mathrm{yr}$. The color scales are $\log _{10} f_{\text {water }}$.
$M_{\mathrm{pl}, 0}=3,5$, and $10 M_{\oplus}$. We stop pebble accretion when $M_{\mathrm{pl}}$ reaches $M_{\text {peb,iso }}$. Because $\alpha$ is smaller for smaller $r_{\mathrm{d}, 0}$ (Eq. (14)) and $h / r$ is lower for smaller $\dot{M}_{\mathrm{g}, 0}$ and higher $\alpha$ (Eq. (3)), $M_{\text {peb,iso }}$ is smaller for smaller $r_{\mathrm{d}, 0}$ and $\dot{M}_{\mathrm{g}, 0}$. The dry regime (red-colored regime) in the left bottom part of the plots represent the cases of $M_{\mathrm{pl}, 0}>M_{\text {peb,iso }}$. Because $t_{\text {snow }}$ is small in the low $\dot{M}_{\mathrm{g}, 0}$ regions, $f_{\text {water }}$ rapidly increases because of a high pebble flux until $M_{\mathrm{pl}}$ increases to $M_{\text {peb,iso }}$. Thereby, the edge in $f_{\text {water }}$ at $M_{\mathrm{pl}, 0} \simeq M_{\text {peb,iso }}$ is sharp.

For the same $t_{\mathrm{diff}}, \dot{M}_{\mathrm{g}, 0}$, and $r_{\mathrm{d}, 0}$ as the above, $M_{\text {peb,iso }} \simeq$

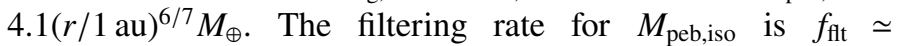
$0.5(r / 1 \mathrm{au})^{1 / 14}$ in 2D case (Eq. (29)). If ice giants or cores of gas giants are formed, even before their mass exceeds $M_{\text {peb,iso }}$, the pebble flux is reduced by $\sim 50 \%$ by individual ice giants.

If Jupiter's core is formed in the outer region, it shuts down the pebble mass flux into the terrestrial planet region. Morbidelli et al. (2016) proposed that the pebble flux truncation by the formation of Jupiter accounts for the dichotomy of our solar system - the total solid mass contained in Jupiter, Saturn, Uranus, and Neptune is about 50 times larger than the total mass of terrestrial planets. Jupiter's core must be formed at $M_{\text {res }} \gtrsim 10 M_{\oplus}$. From Eq. (47) with $M_{\mathrm{d}, 0} \sim 10^{-8} M_{\odot} \mathrm{yr}^{-1}$ and $t_{\mathrm{diff}} \sim 3 \times 10^{6} \mathrm{yr}$, $M_{\text {res }}(t) \sim 200\left(t_{\mathrm{pff}} / t\right)^{\gamma} M_{\oplus}$, which is $M_{\text {res }}(t) \sim 20\left(t / 10^{6} \mathrm{yr}\right)^{-1.5} M_{\oplus}$ for $r_{\mathrm{d}, 0} \sim 100 \mathrm{au}$. Hence, the Jupiter's core formation time $t_{\text {jup }}$

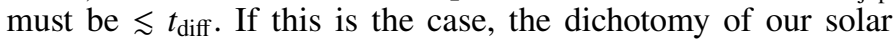
system could be created. However, the water fraction of the 

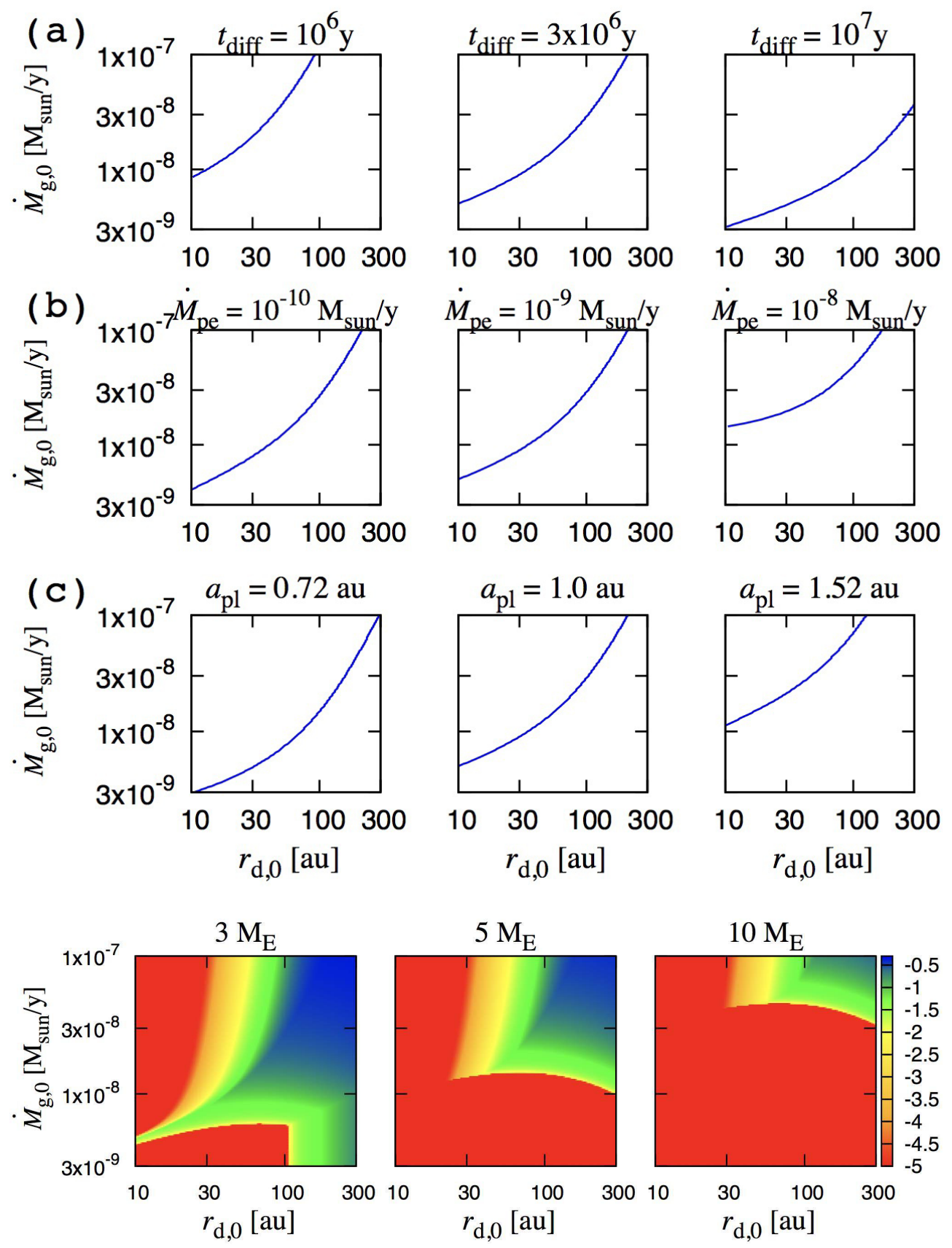

Fig. 13. Condition of $t_{\text {snow }}=10 t_{\text {pff }}$ as a function of $r_{\mathrm{d}, 0}$ and $\dot{M}_{\mathrm{g}, 0}$. Panels $a$ : dependence on the disk diffusion

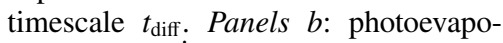
ration rate $\left(\dot{M}_{\mathrm{pe}}\right)$. Panels $c$ : planetary orbital radius $\left(a_{\mathrm{pl}}\right)$. The right regions from the curves represent water-rich regions $\left(f_{\text {water }} \sim 1 / 2\right)$. In the panels a, we used $\dot{M}_{\mathrm{pe}}=10^{-9} M_{\odot} \mathrm{yr}^{-1}$ and $a_{\mathrm{pl}}=1 \mathrm{au}$, $t_{\text {diff }}=3 \times 10^{6} \mathrm{yr}$ and $a_{\mathrm{pl}}=1 \mathrm{au}$ in the panels b, and $\dot{M}_{\mathrm{pe}}=10^{-9} M_{\odot} \mathrm{yr}^{-1}$ and $t_{\text {diff }}=3 \times 10^{6} \mathrm{yr}$ in the panels $\mathrm{c}$. terrestrial planets is a problem in this case, in addition to a problem of too fast type I migration of the core (Matsumura et al. 2017). Our results show that $f_{\text {water }}$ rapidly increases after $t=t_{\text {snow }}$ and becomes saturated before $t \sim t_{\text {diff }}$. If $t_{\text {snow }}>t_{\text {jup }}, f_{\text {water }}=0$ for terrestrial planets. Otherwise, it is likely that $f_{\text {water }}$ is already close to the saturated value because the increase of $f_{\text {water }}$ is very rapid. It is difficult for giant planet formation to directly produce modestly low values of water mass fraction $\left(\sim 10^{-4}-10^{-2}\right)$ corresponding to the Earth and ancient Mars. The modestly low values are attained by disk parameters with $t_{\text {snow }} \sim 10 t_{\text {pff }}$ as we showed.

We also point out that $\mathrm{D} / \mathrm{H}$ ratio is expected to be radially uniform among the Earth, asteroids, and comets if water is delivered by icy pebbles that form in disk outer regions and drift all the way to the host star. However, observations show that in our solar system, D/H ratios of Oort cloud comets are clearly higher than the Earth (e.g., Marty 2012). One possibility to reconcile the discrepancy is the shutdown of the pebble flux by Jupiter formation (Kruijer et al. 2017). Because pebble formation front migrates outward, the isotope ratios of drifting pebbles before and after Jupiter formation, which are the building materials for terrestrial planets and those for comets, respectively, should be different. A more careful comparison should be necessary between planet formation model and cosmo-chemical data.

\section{Summary}

If water is not delivered to rocky planets in HZs, the planets cannot be actual habitats because $\mathrm{H}_{2} \mathrm{O}$ ice condenses in the disk regions well beyond the HZs. In the pebble accretion model, accretion of icy pebbles after the snowline passage may be a primary mechanism to deliver water to the rocky planets.

In this paper, we have investigated the water delivery to rocky planets by pebble accretion around solar-type stars through a 1D simulation of the growth of icy dust grains to pebbles and the pebble radial drift in an evolving disk. We assume that the 
planetary embryos did not migrate significantly and consist of pure rock, which means that accretion of ice starts when the snowline migrates inward and passes the planetary orbit due to disk evolution. Our previous paper, Sato et al. (2016), pointed out that the water fraction of the final planets are determined by the timings of the snowline passage through the planetary orbit $\left(t=t_{\text {snow }}\right)$ and disk gas depletion because pebble accretion is fast and efficient. While Sato et al. (2016) used a simple static disk model, we used the evolving disk model due to viscous diffusion based on the self-similar solution with constant viscous $\alpha$ (Lynden-Bell \& Pringle 1974) and simultaneously calculated pebble formation/drift/accretion and snowline migration with the disk model. Because the snowline migration is correlated with global disk diffusion in the evolving disk model, we found that for a water fraction of the final planets $\left(f_{\text {water }}\right)$, the snowline passage time $\left(t_{\text {snow }}\right)$ relative to the time $\left(t_{\text {pff }}\right)$ at which pebble formation front reaches the disk outer edge is more important than that relative to the disk gas depletion timescale. Our simulation shows that the ice dust mass $\left(M_{\text {res }}\right)$ preserved in the disk outer region at $t=t_{\text {snow }}$ determines the water fraction of the final planets. The accreted ice mass to the planet is estimated by $\sim(1 / 2) f_{\mathrm{flt}} M_{\text {res }}$, where the filtering factor $f_{\mathrm{flt}}$ is the fraction of the pebble mass flux that is accreted onto the planet. Because $M_{\text {res }}$ rapidly decreases after $t \sim t_{\mathrm{pff}}, t_{\mathrm{snow}} / t_{\mathrm{pff}}>10$ or $<10$ is crucial for

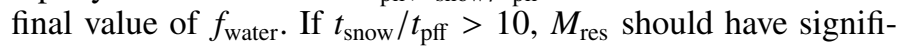
cantly decayed when icy pebble accretion starts at $t=t_{\text {snow }}$.

Using these numerical results, we derived an analytical formula for $f_{\text {water }}$ by icy pebble accretion. In the formula, $f_{\text {water }}$ is explicitly given as a function of the ratio $t_{\text {snow }} / t_{\text {pff }}$ and the disk parameters. The parameter $t_{\mathrm{snow}} / t_{\mathrm{pff}}$ is also determined by the disk parameters. As a result, $f_{\text {water }}$ is predicted by the disk parameters, especially the initial disk mass accretion rate $\dot{M}_{\mathrm{g}, 0}$ and initial disk size $r_{\mathrm{d}, 0}$. It is insensitive to pebble accretion parameters such as the planet mass and Stokes number of drifting pebbles.

We found that the expected water fraction of an Earth ana$\log$ near 1 au has $f_{\text {water }} \sim 10^{-4}-10^{-2}$, which may correspond to the value of the current Earth, in disks with an initial disk size of $r_{\mathrm{d}, 0} \sim 30-50 \mathrm{au}$ and the initial disk mass accretion rate $\dot{M}_{\mathrm{g}, 0} \sim\left(10^{-8}-10^{-7}\right) M_{\odot} \mathrm{yr}^{-1}$. For $\dot{M}_{\mathrm{g}, 0} \gtrsim 2 \times 10^{-8} M_{\odot} \mathrm{yr}^{-1}$, both the Earth and Mars analogs have $f_{\text {water }} \sim 10^{-4}-10^{-2}$, while $f_{\text {water }}$ is generally larger for Mars than for Earth. Because these disks may be median or slightly compact/massive disks among classical T Tauri stars, our results suggest that rocky planets in HZs in exoplanatery systems around solar-type stars often have a water fraction similar to the Earth if pebble accretion is responsible for water delivery, while large diversity in the water fraction due to the variations of disk conditions may also exist.

Acknowledgements. We thank Michiel Lambrechts for detailed and helpful comments. This work was supported by JSPS KAKENHI 15H02065 and 16K1766 and by MEXT KAKENHI 18H05438.

\section{References}

Armitage, P. J., Simon, J. B., \& Martin, R. G. 2013, ApJ, 778, L14 Ataiee, S., Baruteau, C., Alibert, Y., \& Benz, W. 2018, A\&A, 615, A110
Bai, X.-N., Ye, J., Goodman, J., \& Yuan, F. 2016, ApJ, 818, 152 Bercovici, D., \& Karato, S.-i. 2003, Nature, 425, 39

Birnstiel, T., Klahr, H., \& Ercolano, B. 2012, A\&A, 539, A148

Bitsch, B., Morbidelli, A., Johansen, A., et al. 2018, A\&A, 612, A30

Brauer, F., Dullemond, C. P., \& Henning, T. 2008, A\&A, 480, 859

Brown, H. 1949, in The Atmospheres of the Earth and Planets, ed. G. P. Kuiper (Chicago: University of Chicago), 258

Clifford, S. M., Lasue, J., Heggy, E., et al. 2010, J. Geophys. Res. Planets, 115 E07001

di Achille, G., \& Hynek, B. M. 2010, Lunar Planet. Sci. Conf., 41, 2366

Donahue, T. M., Hoffman, J. H., Hodges, R. R., \& Watson, A. J. 1982, Science, 216, 630

Fei, H., Yamazaki, D., Sakurai, M., et al. 2017, Sci. Adv., 3, e1603024

Garaud, P., \& Lin, D. N. C. 2007, ApJ, 654, 606

Genda, H., \& Abe, Y. 2005, Nature, 433, 842

Greenwood, J. P., Karato, S.-i., Vander Kaaden, K. E., Pahlevan, K., \& Usui, T. 2018, Space Sci. Rev., 214, 92

Guillot, T., Ida, S., \& Ormel, C. W. 2014, A\&A, 572, A72

Gundlach, B., \& Blum, J. 2015, ApJ, 798, 34

Haisch, Jr. K. E., Lada, E. A., \& Lada, C. J. 2001, ApJ, 553, L153

Hartmann, L., Calvet, N., Gullbring, E., \& D’Alessio, P. 1998, ApJ, 495, 385

Hartmann, L., Herczeg, G., \& Calvet, N. 2016, ARA\&A, 54, 135

Hasegawa, Y., Okuzumi, S., Flock, M., \& Turner, N. J. 2017, ApJ, 845, 31

Hirose, S., \& Turner, N. J. 2011, ApJ, 732, L30

Hirschmann, M. M. 2006, Ann. Rev. Earth Planet. Sci., 34, 629

Ida, S., Guillot, T., \& Morbidelli, A. 2016, A\&A, 591, A72

Johansen, A., Ida, S., \& Brasser, R. 2019, A\&A, 622, A202

Klahr, H., Pfeil, T., \& Schreiber, A. 2018, Handbook of Exoplanets (London: Springer), 138

Kruijer, T. S., Burkhardt, C., Budde, G., \& Kleine, T. 2017, Proc. Nat. Acad. Sci., 114,6712

Kurokawa, H., Sato, M., Ushioda, M., et al. 2014, Earth Planet. Sci. Lett., 394, 179

Lambrechts, M., \& Johansen, A. 2012, A\&A, 544, A32

Lambrechts, M., \& Johansen, A. 2014, A\&A, 572, A107

Lambrechts, M., Johansen, A., \& Morbidelli, A. 2014, A\&A, 572, A35

Lunine, J. I., Chambers, J., Morbidelli, A., \& Leshin, L. A. 2003, Icarus, 165, 1

Lynden-Bell, D., \& Pringle, J. E. 1974, MNRAS, 168, 603

Lyra, W., \& Umurhan, O. 2018, PASP, submitted

Machida, R., \& Abe, Y. 2010, ApJ, 716, 1252

Marty, B. 2012, Earth Planet. Sci. Lett., 313, 56

Matsumura, S., Brasser, R., \& Ida, S. 2016, ApJ, 818, 15

Matsumura, S., Brasser, R., \& Ida, S. 2017, A\&A, 607, A67

Min, M., Dullemond, C. P., Kama, M., \& Dominik, C. 2011, Icarus, 212, 416

Morbidelli, A., Chambers, J., Lunine, J. I., et al. 2000, Meteorit. Planet. Sci., 35 , 1309

Morbidelli, A., Bitsch, B., Crida, A., et al. 2016, Icarus, 267, 368

Mori, S., Bai, X.-N., \& Okuzumi, S. 2019, ApJ, 872, 98

Musiolik, G., Teiser, J., Jankowski, T., \& Wurm, G. 2016, ApJ, 818, 16

Nomura, R., Hirose, K., Uesugi, K., et al. 2014, Science, 343, 522

O'Brien, D. P., Walsh, K. J., Morbidelli, A., Raymond, S. N., \& Mandell, A. M. 2014, Icarus, 239, 74

Oka, A., Nakamoto, T., \& Ida, S. 2011, ApJ, 738, 141

Okuzumi, S., \& Tazaki, R. 2019, ApJ, submitted

Okuzumi, S., Tanaka, H., Kobayashi, H., \& Wada, K. 2012, ApJ, 752, 106

Ormel, C. W. 2014, ApJ, 789, L18

Ormel, C. W., \& Klahr, H. H. 2010, A\&A, 520, A43

Ormel, C. W., \& Kobayashi, H. 2012, ApJ, 747, 115

Raymond, S. N., Quinn, T., \& Lunine, J. I. 2004, Icarus, 168, 1

Sato, T., Okuzumi, S., \& Ida, S. 2016, A\&A, 589, A15

Shakura, N. I., \& Sunyaev, R. A. 1973, A\&A, 24, 337

Suzuki, T. K., Ogihara, M., Morbidelli, A., Crida, A., \& Guillot, T. 2016, A\&A, 596, A74

Takeuchi, T., \& Lin, D. N. C. 2005, ApJ, 623, 482

Wada, K., Tanaka, H., Suyama, T., Kimura, H., \& Yamamoto, T. 2009, ApJ, 702, 1490

Williams, J. P., \& Cieza, L. A. 2011, ARA\&A, 49, 67

Youdin, A. N., \& Lithwick, Y. 2007, Icarus, 192, 588 